\title{
Theoretical and Numerical Study of Several Free Molecular Flow Problems
}

\author{
Chunpei Cai* \\ ZONA Technology Inc., Scottsdale, Arizona, 85258 \\ Iain D. Boyd ${ }^{\dagger}$ \\ University of Michigan, Ann Arbor, Michigan, 48109
}

\begin{abstract}
In this study, by using several velocity-position relations, we investigate free molecular effusion flows out of exits with a zero or nonzero average exit velocity. This study yields six sets of exact solutions and expressions for number density and velocity distributions in front of the exits. These solutions include the exact exit geometry parameters. Several properties of the solutions are discussed mathematically and physically. Numerical simulation results validate the analytical solutions. In general, the comparisons between analytical and numerical results are virtually identical.
\end{abstract}

\section{Introduction}

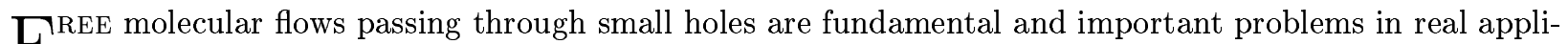
F cations, such as materials processing in high vacuum chambers, ${ }^{1}$ spacecraft design, ${ }^{2}$ and metrology of gas flow. ${ }^{3}$ Several investigators have proposed analytical expressions for the mass flow rate near the free-molecular regime in the case of outflow from an orifice into vacuum, $, 5,6,7,8,9$ which are valid in a very small range of gas rarefaction. Recently, several simulations of rarefied gas flow through a thin orifice ${ }^{10}$ were reported.

In aerospace engineering, plume flows exhausted from electric propulsion devices are essentially free molecular flow. This study is an initial attempt to analytically investigate the plume flows from electric propulsion systems. Electric propulsion systems, such as Hall thrusters, have several merits over traditional chemical thrusters and they are designed to operate in space for purposes of primary propulsion and on-orbit applications such as station keeping. For electric thrusters, their plume flows are of major interest because of several reasons. First, plume flows can be used to study the performance of a thruster; second, plume impingement onto a spacecraft can have significant adverse effects. The plume impingement is usually caused by the Charge Exchange(CEX) effect. When a fast ion collides with a slow neutral, one or two electrons may transfer from the slow neutral to the fast ion and result in a fast neutral and a slow ion. Under the electric field effect, this slow ion can drift to large plume angles where sensitive surfaces such as solar cells may be located. Impingement and deposition on the sensitive surfaces may result in eventual failure of the devices. Hence, plume flows are always important research topics for development of electric propulsion thrusters.

There are many experimental measurements and numerical simulations of plasma plumes of electric thrusters, however, there are very few reports of analytical studies in the literature. Most related previous kinetic work in the literature was concentrated on free molecular flows out of a simplified geometry such as an orifice. ${ }^{5}$ Obviously, further analytical results of the plume flow field with more detailed geometries can provide insights and aid in the understanding of the plume flows out of electric thrusters.

${ }^{*}$ CFD Specialist, 8489 E.Ironword Square Dr., previously graduate student research assistant, Department of Aerospace Engineering, the University of Michigan, Ann Arbor, Michigan. AIAA member.

†Professor, Department of Aerospace Engineering, 1320 Beal Ave., AIAA Associate Fellow. 
Although there are several fundamental aspects of a plume flow out of an electric thruster, this study considers only the primary features. The fundamental features are: First, most electric plume flows are almost free molecular. The mean free path for gas propellant out of a thruster can be estimated with the following hard sphere equation:

$$
\lambda=\frac{1}{\sqrt{2} \pi d^{2} n_{0}}
$$

With a typical number density of $5 \times 10^{18} \mathrm{~m}^{-3}$ at a thruster exit, the mean free path for xenon is $0.268 \mathrm{~m}$, which is wider than a typical Hall thruster acceleration channel. Second, the average velocity at a thruster exit plane is greater than zero. The average neutral speed at the thruster exit plane is usually sonic, and the average ion speed is always supersonic due to electric acceleration inside the acceleration channel. Third, electric field effects in the plume are important for ions. As an initial effort, by neglecting the electric field and all collision effects, this paper investigates six different free molecular gas flows out of an exit with different geometries. The exact solutions to the six problems provide first order approximations to cold plume flows, and in a future study, electric field effects and collision effects can be included based on the analytical solutions. Further, in this study, most discussions are concentrated on effusion problems with zero exit velocities, which simplifies the situation and provides a solid basis and insights for further study.

In the rest of this study, section II presents the problems to be studied and a general solution method; sections III-VIII present six free molecular flow problems, their analytical solutions, discussions of their properties or simplifications of the solutions, and comparisons with particle simulation results; section IX summarizes this study.

\section{Free Molecular Flow Problems and General Treatment}

The flows considered in this study are six different problems of free molecular gas flows out of an exit into vacuum with a fixed average speed. The geometry configurations for the exit and the average exit speed are different:

(1) A two-dimensional thin slit with a width of $2 H$, average exit velocity $U_{0}$ is zero.

(2) A rectangular slit with a width of $2 L$ and a height of $2 H$, average exit velocity $U_{0}$ is zero.

(3)A slit formed by two concentered rectangles with widths and heights $2 L$ by $2 H$, and $2 L_{1}$ by $2 H_{1}$, average exit velocity $U_{0}$ is zero.

(4) A circular exit characterized by a radius of $R$, average exit velocity $U_{0}$ is zero.

(5) An annulus characterized by an inner radius $R_{1}$ and an outer radius $R_{2}$, average exit velocity $U_{0}$ is zero.

(6) A two-dimensional thin slit with a width of $2 H$, average exit velocity $U_{0}$ is greater than zero.

This study adopts the following coordinate systems: Denote the plume direction as the $\mathrm{X}$-axis direction, the direction normal to the $\mathrm{X}$-axis as the $\mathrm{Y}$-axis direction, and the middle point of the slit/circle center/annulus center is the origin. The objective of this study is to obtain the analytical plume field flow solutions, especially the number density and velocities at any point downstream of the exit. Suppose the gas at the exit is in equilibrium with a uniform static temperature $T_{0}$ and a uniform number density $n_{0}$.

\section{A. General Methods}

For a dilute gas flow at rest in equilibrium, the velocity distribution is described as a full Maxwellian distribution. ${ }^{11,12,13}$ With a total number density $n_{0}$ and a temperature $T_{0}$ the velocity distribution function at any point follows a Maxwellian distribution:

$$
f(u, v, w)=n_{0}\left(\frac{\beta}{\pi}\right)^{3 / 2} \exp \left(-\beta\left(u^{2}+v^{2}+w^{2}\right)\right)
$$


where $\beta=\frac{1}{2 R T_{0}}$. The highest velocity probability occurs at phase point $(u, v, w)=(0,0,0)$. For a velocity with a nonzero average value of $U_{0}$ along the $X$ direction but a zero average value along the $Y$ and $Z$ directions, the distribution Equation 2 is shifted along the u-axis by a value of $U_{0}$.

With a known velocity distribution $f(u, v, w)$ at a point $(X, Y, Z)$, the average number density normalized by a reference number density and velocities at $(X, Y, Z)$ can be evaluated using the velocity distribution function: ${ }^{12}$

$$
\begin{gathered}
n(X, Y, Z)=\int_{\Omega} f(u, v, w) d u d v d w \\
U(X, Y, Z)=\frac{1}{n(X, Y, Z)} \int_{\Omega} u f(u, v, w) d u d v d w \\
V(X, Y, Z)=\frac{1}{n(X, Y, Z)} \int_{\Omega} v f(u, v, w) d u d v d w \\
W(X, Y, Z)=\frac{1}{n(X, Y, Z)} \int_{\Omega} w f(u, v, w) d u d v d w
\end{gathered}
$$

Hence, the critical step is to obtain the velocity distribution function and the integral domain in the velocity phase space. Narasimha observed an important fact when studying a free molecular flow of gas escaping from an orifice: ${ }^{5}$

Observation: For any point $(X, Y, Z)$ in front of the slit, its velocity space can only have non-zero velocity within a specific solid angle $\Omega$ subtended by the specific point and exit edges:

$$
f(u, v, w)= \begin{cases}n_{0}\left(\frac{\beta}{\pi}\right)^{3 / 2} \exp \left(-\beta\left(u^{2}+v^{2}+w^{2}\right)\right), & u, v, w \in \Omega \\ 0, & u, v, w \notin \Omega .\end{cases}
$$

where $\beta=\frac{1}{2 R T_{0}}$ and $\Omega$ represents the integral domain in the velocity phase space.

Although this observation is critical, to truly attack the free molecular flows listed in this study, we believe it is necessary to reinterpret this fact with a deeper view as a velocity-position relation: From a given point on the exit, only particles with specific velocity components can arrive at a specific point $(X, Y, Z)$ in front of the exit. This relation takes different forms in the six cases and will be illustrated later as Equations 8, 15, 36 and 52. These equations actually have three effects: First they guarantee a one-to-one mapping relation between velocity phase spaces and provide a complete support to Equation 7; Second, they can provide boundaries for the integral domain; Third, they may simplify the integral process by changing integral variables from velocities to geometry parameters of the exit.

In this study, many integral formulas are used, several complex integrals and complex derivations can be found in one of the References. ${ }^{14}$

\section{Problem One: Free Molecular Effusion Flow from a Thin Slit with a Zero Average Velocity $\left(U_{0}=0\right)$}

\section{A. Analytical Results}

In this case, the previous velocity-position relation takes the following format as Equation 8: from a point $(0, h)$ on the slit, particles can reach the point $(X, Y)$ if their two velocity components $(u, v)$ satisfy the following relation:

$$
\tan (\theta)=\frac{Y-h}{X}=\frac{v}{u},-H<h<H
$$

On the other hand, particles from point $(0, h)$ cannot arrive at the point $(X, Y)$ if their two velocity components do not satisfy the above relation. Hence, a correct velocity component relation is the necessary and sufficient condition that a particle can travel from a point on the exit to a specific point in front of the exit.

3 of 33

American Institute of Aeronautics and Astronautics 
This important relation shows that there is a one to one mapping relation between velocity space for the point on the slit and the velocity space of the point $(X, Y)$. A slice of the velocity space for point $(0, h)$ is strictly mapped onto the velocity space for point $(X, Y)$. There is neither loss nor gain during the mapping. Figure 1 illustrates the velocity spaces for two points in front of the exit. One point, A, is outside the plume core region, while the other point, $\mathrm{B}$, is inside the plume core. Velocity space "A1-O-A2" represents the first point's integral domain while "B1-O-B2" represents the second point's integral domain. Line O-A3 represents the velocity contributed by particles starting from point 3, while the particle starting at point 3 that can arrive at point $\mathrm{B}$ is represented by the positive $\mathrm{u}$-axis.
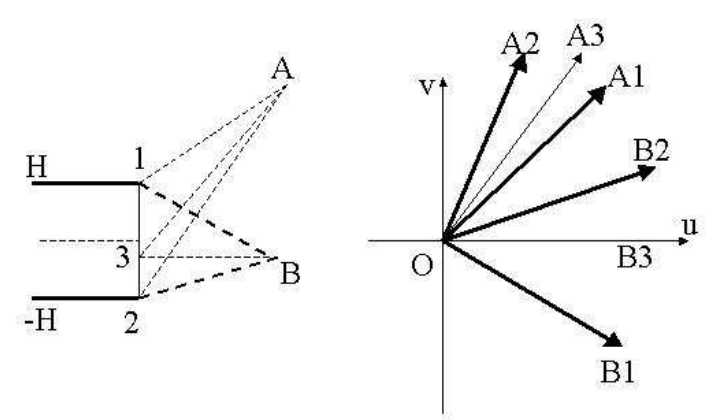

Figure 1. Velocity Spaces for Points Inside or Outside Plume Core.

The non-zero values in the velocity space of point $(X, Y)$ form a pie shape bounded by two lines from the slit edge points to the point $(X, Y)$, and the number density and velocities at the point $(X, Y)$ can be obtained by integrating the probability distribution function within the two lines:

$$
\tan \left(\theta_{1}\right)=\frac{Y-H}{X}, \tan \left(\theta_{2}\right)=\frac{Y+H}{X}
$$

With this simple integral domain in the velocity phase space, the integrals can be simplified with the following relation:

$$
d u d v=\bar{V} d \bar{V} d \theta
$$

where $\bar{V}=\sqrt{u^{2}+v^{2}}$.

With this relation, the expressions for the number density and the velocities at point $(X, Y)$ are:

$$
\begin{gathered}
n(X, Y)=\int_{\Omega} \frac{\beta}{\pi} \exp \left(-\beta\left(u^{2}+v^{2}\right)\right) d u d v=\frac{\theta_{2}-\theta_{1}}{2 \pi} \\
\frac{U(X, Y)}{\sqrt{2 R T_{0}}}=\frac{\sqrt{\pi}\left(\sin \theta_{2}-\sin \theta_{1}\right)}{2\left(\theta_{2}-\theta_{1}\right)} \\
\frac{V(X, Y)}{\sqrt{2 R T_{0}}}=\frac{\sqrt{\pi}\left(\cos \theta_{1}-\cos \theta_{2}\right)}{2\left(\theta_{2}-\theta_{1}\right)}
\end{gathered}
$$

$$
4 \text { of } 33
$$


From the above results, it is informative to obtain the exact solutions of properties along the centerline, which is important to many applications. Along the centerline $Y=0$, where $\theta_{1}=-\theta_{2}$ :

$$
n(X, 0)=\frac{1}{\pi} \arctan \left(\frac{H}{X}\right), \frac{U(X, 0)}{\sqrt{2 R T_{0}}}=\frac{\sqrt{\pi} \sin (\arctan (H / X))}{2 \arctan (H / X)}, V(X, 0)=0
$$

When $X$ increases from 0 to infinity, the number density decreases from $\frac{1}{2}$ to 0 and the speed ratio increases from $\frac{1}{\sqrt{\pi}}$ to $\frac{\sqrt{\pi}}{2}$. It should be pointed out that this speed ratio is obtained by a thermal speed characterized by the temperature at the slit, not the local temperature.

Several properties of 11-13 are:

1. Flow symmetries are preserved: $n(X,-Y)=n(X, Y), U(X,-Y)=U(X, Y), V(X,-Y)=-V(X, Y)$.

2. On the slit where $X=0,-H<Y<H:-\theta_{1}=\theta_{2}=\frac{\pi}{2}, n(0, Y)=\frac{1}{2}, V(0, Y)=0, U(0, Y)=$ $\sqrt{2 R T_{0} / \pi}$. The nonzero velocity along the $X$ direction does not contradict the zero mean velocity distribution boundary condition at the slit.

3. At locations above the slit: $X=0, Y>H: \theta_{1}=\theta_{2}=\frac{\pi}{2}$, the following relations hold: $n(0, Y)=0$, $V(0, Y)=0, U(0, Y)=0$. Hence, the two edge-points are discontinuity points because of abrupt changes of number density.

4. The mass flux relation through the slit: $n(0, Y)=\frac{1}{2}$ and $U(0, Y)=\sqrt{2 R T_{0} / \pi}$ for $-H<Y<H$, $-\theta_{1}=\theta_{2}=\frac{\pi}{2}$, hence the mass flow rate out of the slit $=\frac{1}{4} m n_{0} \sqrt{8 R T_{0} / \pi}$, which is a well-known kinetic flow result.

5. On the exit tip point: $n(0, H)=\frac{1}{4}=\frac{1}{2} n(0,0), U(0, H)=V(0, H)=\sqrt{2 R T_{0} / \pi}=U(0,0)$. Obviously, number density and velocities along the vertical line passing through the exit tip are not continuous.

6. Depending on the distance from point $(X, Y)$ to the origin, the flow field can be classified into four regions: near field and inside core region, near field and outside core region, far field inside core region and far field outside core region. The last region is the largest where the slit effects are quite minor, and the velocities in this region have a relation $U(X, Y) / V(X, Y) \propto /\left(\theta_{1}+\theta_{2}\right) \approx 1 / \alpha$, where $\alpha$ is the angle subtended from the point to the two exit edge points. This relation indicates that the streamlines in that region are almost straight.

\section{B. DSMC Simulation and Discussions}

The above set of analytical solutions is validated by comparisons with a numerical simulation. Because the flows are free molecular, the direct simulation Monte Carlo (DSMC) method of Bird ${ }^{11}$ is ideal to provide accurate solutions and gaseous xenon flows out of a slit with a semi-height $H=0.1 \mathrm{~m}$. In the simulation, the number density at the slit is set to a value corresponding to $K n=20$ with a characteristic length $2 H=0.2$ $\mathrm{m}$. To compare with the analytical results, further all DSMC simulations in this study are performed by turning off the collision functions.

The simulation domain used for this case is a square with a side length of 2 meters. There are three typess of boundary conditions in these simulations: the bottom line is a symmetric boundary condition; at the bottom of the left side, there is an inlet boundary condition where particles are introduced into the simulation and their thermal velocities are characterized by a temperature $T_{0}=300 \mathrm{~K}$; the other remaining boundaries are outlet boundary conditions and when particles cross these sides, they are removed from the simulation. A mesh of 75 by 60 cells along the $\mathrm{X}$-axis and $\mathrm{Y}$-axis directions is adopted. The main reason for adopting such a dense mesh is to provide high spatial resolution for comparison with the analytical results.

This study adopts a specific DSMC simulation package named $\mathrm{MONACO}^{15}$ to perform this simulation. Approximately one million particles are employed in the final sampling stage for this case. The simulation takes approximately 30 hours on a parallel machine with two processors.

\section{5 of 33}

American Institute of Aeronautics and Astronautics 


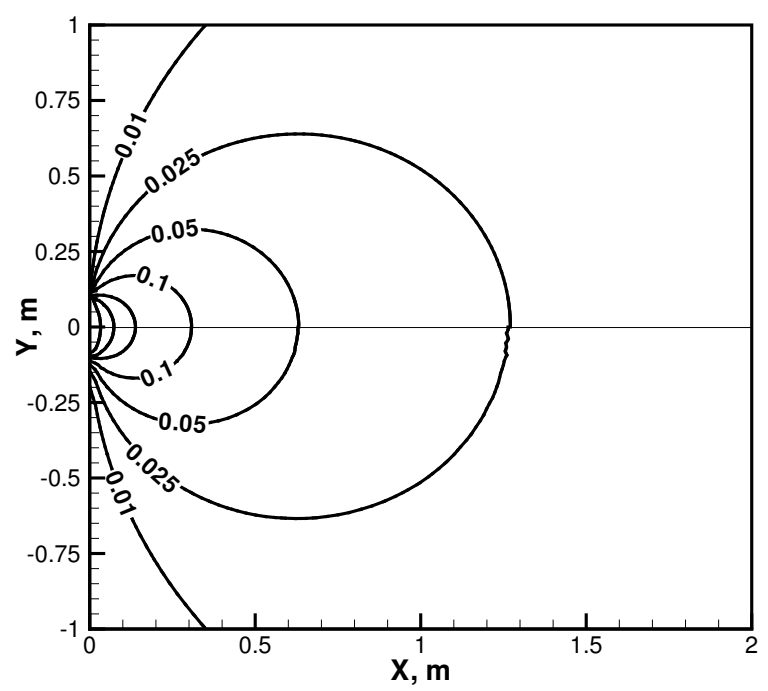

Figure 2. Case 1: Contours of Number Density $\left(H=0.1 m, U_{0}=\mathbf{0}\right.$, Top: Analytical, Bottom: DSMC without Collisions).

Figure 2 shows a comparison of number density contours obtained with the analytical results and the DSMC simulation. The average exit velocity $U_{0}$ is set to zero. Figures 3 and 4 show two comparisons of velocity contours along the $\mathrm{X}$ and $\mathrm{Y}$ directions. The comparison indicates that for this true effusion problem these results are almost identical. Both theoretical and numerical results indicate that there is an exit effect dominating the area close to the exit, while in the far field, the velocity contours are essentially straight lines. Notice that in Equations 12 and 13, the denominator is zero along the vertical line $X=0, Y>H$, hence it is a singularity line. The accumulation of contour lines along this singularity line, shown in the analytical contour results of $V$, is not physical.

Figure 5 shows comparisons between the analytical results and the simulation results of velocity and number density distributions along lines $Y=0$ and $Y=H$. It clearly confirms the previous discussion that the velocity at the slit center and the tip point are the same but the number density at the tip point is only half of the value at the slit center.

\section{Problem Two: Free Molecular Effusion Flow From a Rectangular Exit 2H by $2 \mathrm{~L}$ with a Zero Average Exit Velocity $\left(U_{0}=0\right)$}

\section{A. Analytical Results}

Suppose there is a rectangular exit, which is characterized by a width of $2 \mathrm{~L}$ and a height of $2 \mathrm{H}$, and the average exit velocity is zero. This problem was previous discussed by Patterson. ${ }^{16}$ Suppose there is a point $(X, Y, Z)$ in front of the exit, where $Y>0$ and $Z>0$, then from any point $(0, y, z)$ on the exit, particles can only arrive at the point $(X, Y, Z)$ if their velocity components follow the relation:

$$
\frac{X}{u}=\frac{Y-y}{v}=\frac{Z-z}{w}
$$

where $X>0, Y>0, Z>0$. 


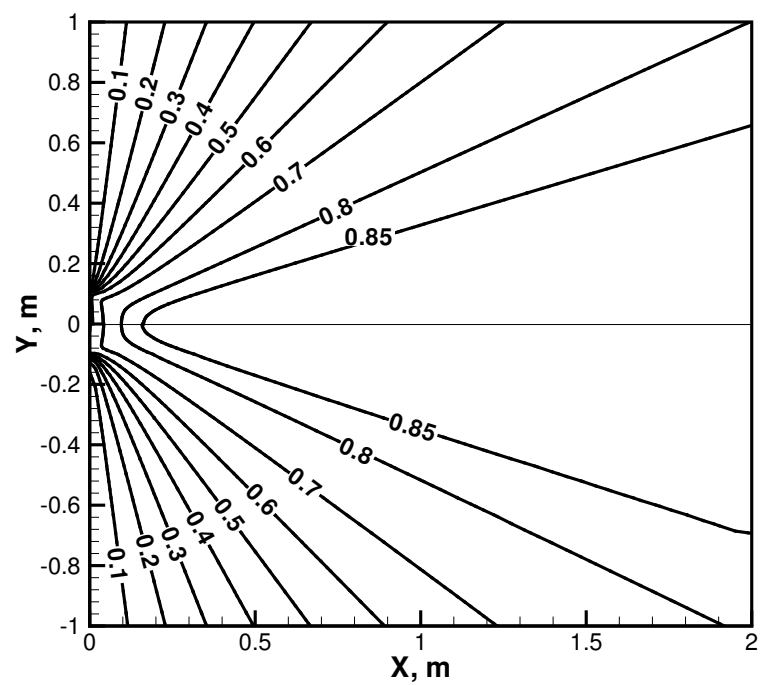

Figure 3. Case 1: Contours of $\frac{U(X, Y)}{\sqrt{2 R T_{0}}}\left(H=0.1 m, U_{0}=\mathbf{0}\right.$, Top: Analytical, Bottom: DSMC without Collisions).

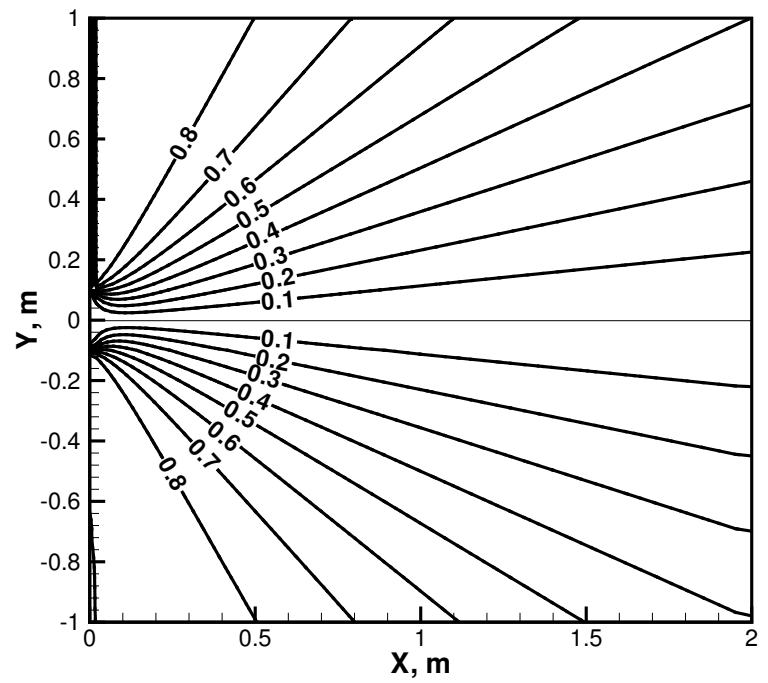

Figure 4. Case 1: Contours of $\frac{V(X, Y)}{\sqrt{2 R T_{0}}}\left(H=0.1 m, U_{0}=\mathbf{0}\right.$, Top: Analytical, Bottom: DSMC without Collisions).

7 of 33

American Institute of Aeronautics and Astronautics 


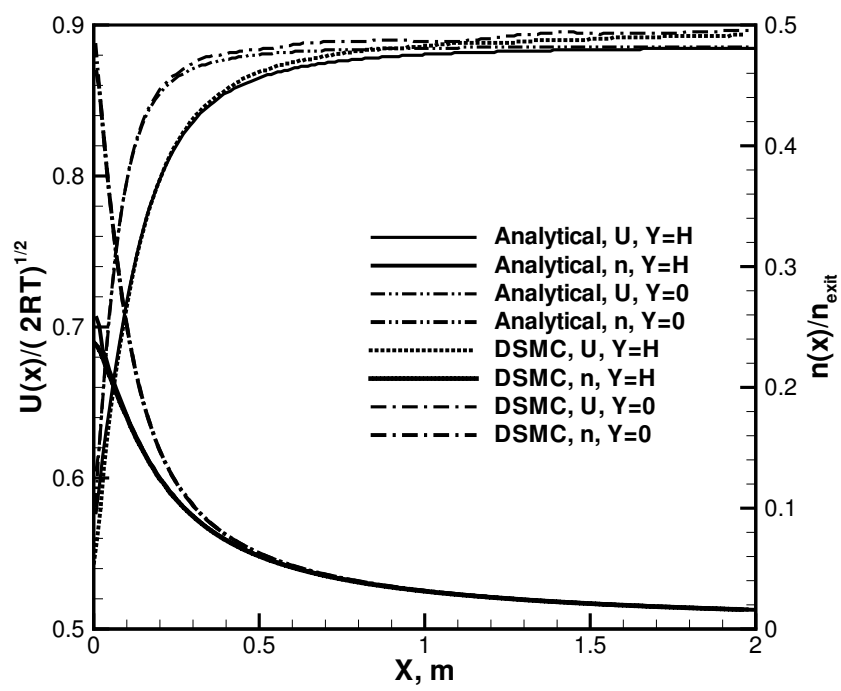

Figure 5. Case 1: Number Density and $\frac{U(X, Y)}{\sqrt{2 R T_{0}}}$ Along $Y=\mathbf{0}$ and $Y=H\left(H=0.1 m, U_{0}=\mathbf{0}\right)$.

From this relation, the number density and velocity integrals can be greatly simplified with the following change of integral variables:

$$
d v d w=\left|\begin{array}{cc}
\frac{\partial v}{\partial y} & \frac{\partial v}{\partial z} \\
\frac{\partial w}{\partial y} & \frac{\partial w}{\partial z}
\end{array}\right| d y d z=\left|\begin{array}{cc}
\frac{-u}{X} & 0 \\
0 & \frac{-u}{X}
\end{array}\right| d y d z=\frac{u^{2}}{X^{2}} d y d z
$$

Obviously with this relation, the rectangular exit edges define an integral domain and the number density and the velocity components can be derived. The final number density result is: ${ }^{14}$

$$
\begin{aligned}
n(X, Y, Z)= & \frac{1}{4 \pi}\left[\arctan \frac{(L-Y)(H-Z)}{X \sqrt{X^{2}+(L-Y)^{2}+(H-Z)^{2}}}+\arctan \frac{(L-Y)(H+Z)}{X \sqrt{X^{2}+(L-Y)^{2}+(H+Z)^{2}}}\right. \\
& \left.+\arctan \frac{(L+Y)(H-Z)}{X \sqrt{X^{2}+(L+Y)^{2}+(H-Z)^{2}}}+\arctan \frac{(L+Y)(H+Z)}{X \sqrt{X^{2}+(L+Y)^{2}+(H+Z)^{2}}}\right]
\end{aligned}
$$

The velocities along the $X, Y, Z$ directions are:

$$
\begin{aligned}
\frac{U(X, Y, Z)}{\sqrt{2 R T_{0}}}= & \frac{1}{4 n \pi \sqrt{\pi}}\left[\frac{H-Z}{\sqrt{(H-Z)^{2}+X^{2}}}\left(\arctan \frac{L-Y}{\sqrt{(H-Z)^{2}+X^{2}}}+\arctan \frac{L+Y}{\sqrt{(H-Z)^{2}+X^{2}}}\right)\right. \\
& +\frac{H+Z}{\sqrt{(H+Z)^{2}+X^{2}}}\left(\arctan \frac{L-Y}{\sqrt{(H+Z)^{2}+X^{2}}}+\arctan \frac{L+Y}{\sqrt{(H+Z)^{2}+X^{2}}}\right) \\
& +\frac{L-Y}{\sqrt{(L-Y)^{2}+X^{2}}}\left(\arctan \frac{H-Z}{\sqrt{(L-Y)^{2}+X^{2}}}+\arctan \frac{H+Z}{\sqrt{(L-Y)^{2}+X^{2}}}\right) \\
& \left.+\frac{L+Y}{\sqrt{(L+Y)^{2}+X^{2}}}\left(\arctan \frac{H-Z}{\sqrt{(L+Y)^{2}+X^{2}}}+\arctan \frac{H+Z}{\sqrt{(L+Y)^{2}+X^{2}}}\right)\right] \\
\frac{V(X, Y, Z)}{\sqrt{2 R T_{0}}=} & \frac{X}{4 n \pi \sqrt{\pi}}\left[\frac{1}{\sqrt{(L-Y)^{2}+X^{2}}}\left(\arctan \frac{H-Z}{\sqrt{(L-Y)^{2}+X^{2}}}+\arctan \frac{H+Z}{\sqrt{(L-Y)^{2}+X^{2}}}\right)\right] \\
& \left.-\frac{1}{\sqrt{(L+Y)^{2}+X^{2}}}\left(\arctan \frac{H-Z}{\sqrt{(L+Y)^{2}+X^{2}}}+\arctan \frac{H+Z}{\sqrt{(L+Y)^{2}+X^{2}}}\right)\right] \\
& 8 \text { of } 33
\end{aligned}
$$

American Institute of Aeronautics and Astronautics 


$$
\begin{aligned}
\frac{W(X, Y, Z)}{\sqrt{2 R T_{0}}}= & \frac{X}{4 n \pi \sqrt{\pi}}\left[\frac{1}{\sqrt{(H-Z)^{2}+X^{2}}}\left(\arctan \frac{L-Y}{\sqrt{(H-Z)^{2}+X^{2}}}+\arctan \frac{L+Y}{\sqrt{(H-Z)^{2}+X^{2}}}\right)\right. \\
& \left.-\frac{1}{\sqrt{(H+Z)^{2}+X^{2}}}\left(\arctan \frac{L-Y}{\sqrt{(H+Z)^{2}+X^{2}}}+\arctan \frac{L+Y}{\sqrt{(H+Z)^{2}+X^{2}}}\right)\right]
\end{aligned}
$$

Note that symmetry is sustained in the above solutions. Suppose $X>0, Y>0$ and $Z>0$ :

$$
\begin{gathered}
n(X, Y, Z)=n(X,-Y, Z)=n(X, Y,-Z)=n(X,-Y,-Z)>0 \\
U(X, Y, Z)=U(X,-Y, Z)=U(X, Y,-Z)=U(X,-Y,-Z)>0 \\
V(X, Y, Z)=-V(X,-Y, Z)=V(X, Y,-Z)=-V(X,-Y,-Z)>0 \\
W(X, Y, Z)=-W(X, Y,-Z)=W(X,-Y, Z)=-W(-X,-Y,-Z)>0
\end{gathered}
$$

Along the exit centerline where $Y=Z=0$ :

$$
\begin{gathered}
n(X, 0,0)=\frac{1}{\pi} \arctan \frac{H L}{X \sqrt{X^{2}+H^{2}+L^{2}}} \\
\frac{U(X, 0,0)}{\sqrt{2 R T_{0}}}=\frac{1}{n \pi \sqrt{\pi}}\left[\frac{H}{\sqrt{H^{2}+X^{2}}} \arctan \frac{L}{\sqrt{X^{2}+H^{2}}}+\frac{L}{\sqrt{X^{2}+L^{2}}} \arctan \frac{H}{\sqrt{X^{2}+L^{2}}}\right] \\
V(X, 0,0)=W(X, 0,0)=0
\end{gathered}
$$

These centerline results contain $H$ and $L$, and are quite different from the standard results of free molecular flow through a small circular orifice. When $X$ changes from 0 to $\infty, n$ changes from $\frac{1}{2}$ to 0 , while $U(0,0,0)=$ $\frac{1}{\sqrt{\pi}}$ with $H=L$.

Another two interesting locations are the rectangle corner and an edge midpoint:

$$
\begin{aligned}
& n(X, L, H)=\frac{1}{4 \pi} \arctan \left(\frac{4 H L}{X \sqrt{X^{2}+4 L^{2}+4 H^{2}}}\right) \\
& \frac{U(X, L, H)}{\sqrt{2 R T_{0}}}=\frac{1}{2 n \sqrt{\pi}^{3}}\left[\frac{H}{\sqrt{4 H^{2}+X^{2}}} \arctan \frac{2 L}{\sqrt{4 H^{2}+X^{2}}}+\frac{L}{\sqrt{4 L^{2}+X^{2}}} \arctan \frac{2 H}{\sqrt{4 L^{2}+X^{2}}}\right] \\
& n(X, 0, H)=\frac{1}{2 \pi} \arctan \frac{2 H L}{X \sqrt{X^{2}+L^{2}+4 H^{2}}} \\
& \frac{U(X, 0, H)}{\sqrt{2 R T_{0}}}=\frac{1}{2 n \sqrt{\pi}^{3}}\left(\frac{2 H}{\sqrt{4 H^{2}+X^{2}}} \arctan \frac{L}{\sqrt{4 H^{2}+X^{2}}}+\frac{L}{\sqrt{L^{2}+X^{2}}} \arctan \frac{2 H}{\sqrt{L^{2}+X^{2}}}\right) \\
& n(X, L, 0)=\frac{1}{2 \pi} \arctan \frac{2 H L}{X \sqrt{X^{2}+4 L^{2}+H^{2}}} \\
& \frac{U(X, L, 0)}{\sqrt{2 R T_{0}}}=\frac{1}{2 n \sqrt{\pi}^{3}}\left(\frac{H}{\sqrt{H^{2}+X^{2}}} \arctan \frac{2 L}{\sqrt{H^{2}+X^{2}}}+\frac{2 L}{\sqrt{4 L^{2}+X^{2}}} \arctan \frac{H}{\sqrt{4 L^{2}+X^{2}}}\right)
\end{aligned}
$$

Immediately, it can be shown that on the exit center and exit tip: $n(0, L, H)=\frac{1}{4} n(0,0,0)=\frac{1}{2} n(0,0, H)$ and $U(0, L, H)=U(0,0,0)$ if $H=L$. Also note the above results we obtained are different from those in one of the references. ${ }^{16}$ The following simulation will validate the results obtained in this paper. 


\section{B. DSMC Simulations and Discussions}

A three-dimensional DSMC simulation is performed to simulate the effusion flow from a rectangular exit with $2 L=2 \times 0.2 \mathrm{~m}$ by $2 H=2 \times 0.2 \mathrm{~m}$. Due to the symmetry in the geometry configuration, only a quarter of the exit is included in the simulation domain. The simulation domain is a cubic shape with side length of 1 meter. A mesh containing 0.5 million tetrahedral cells is used to represent the whole domain, and in the final sampling state, about 6 million particles are maintained. Again, the collision function in MONACO is turned off.

Figure 6 shows the number density contours in the plane $Y=0$ : the solid contours are the analytical results obtained in this study while the dashed contours are the DSMC results. At the exit, both the analytical and DSMC results yield the same value of $\frac{1}{2}$. Figures 7 and 8 show contours of $U(X, 0, Z)$ and $W(X, 0, Z)$ in the plane $\mathrm{Y}=0$. In general, both comparisons are quite satisfactory. In all of these results, the exit effects are well captured. The contours of number density and velocities in the plane $Z=0$ have similar patterns so they are omitted here.

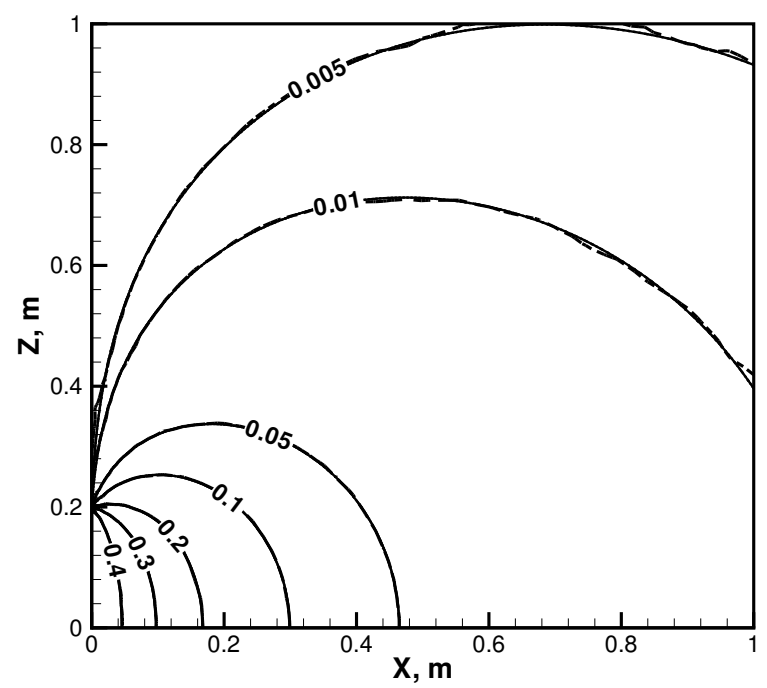

Figure 6. Case 2: Contours of Number Density ( $H=L=0.2 m, U_{0}=\mathbf{0}$, Solid: Analytical, Dashed: DSMC without Collisions).

In addition to the contours, comparisons of properties along the $X$ direction can provide more informative results. Figure 9 shows profiles along one horizontal line passing through the exit corner $(Y=L, Z=H)$. In general, the number density and velocity profiles fit quite satisfactorily. It is also noted that the number density at $X=0$ is close to $\frac{1}{8}$ instead of $\frac{1}{2}$ as predicted by Equation 24 . This is physically correct, because any point inside the domain has a full neighborhood of $2 \pi$, but on the corner, only $\frac{\pi}{2}$ is available. It is expected that the points on an edge but not on a corner will have a density value of $\frac{1}{4}$. Correspondingly, from Equation 24, it is possible to prove that the corner point velocity in the $\mathrm{X}$ direction has the same value as the exit center point. 


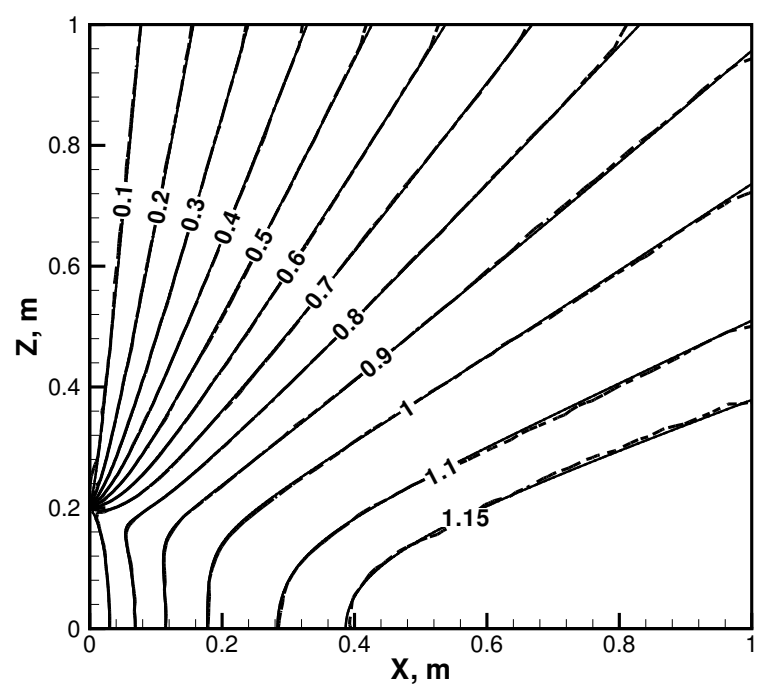

Figure 7. Case 2: Contours of $\frac{U(X, 0, Z)}{\sqrt{2 R T_{0}}}\left(H=L=0.2 m, U_{0}=\mathbf{0}\right.$, Solid: Analytical, Dashed: DSMC without Collisions).

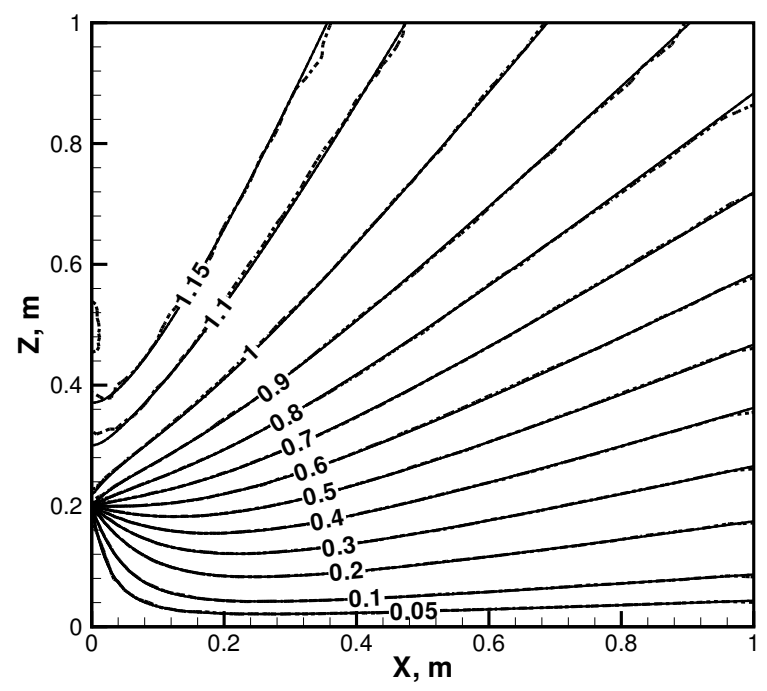

Figure 8. Case 2: Contours of $\frac{W(X, 0, Z)}{\sqrt{2 R T_{0}}}\left(H=L=0.2 m, U_{0}=\mathbf{0}\right.$, Solid: Analytical, Dashed: DSMC without Collisions).

11 of 33

American Institute of Aeronautics and Astronautics 


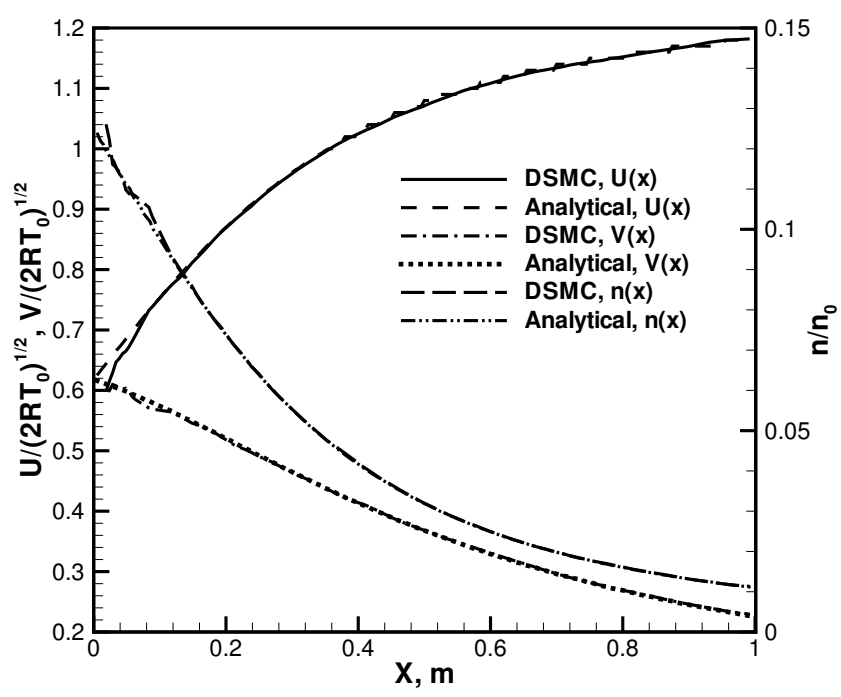

Figure 9. Case 2: Profiles of $\frac{U(X, L, H)}{\sqrt{2 R T_{0}}}$ and $n(X, L, H)\left(H=L=0.2 m, U_{0}=\mathbf{0}\right)$.

\section{Problem Three: Free Molecular Effusion Flow From a Concentered Rectangular Exit $2 \mathrm{H}$ by $2 \mathrm{~L}$ and $2 H_{1}$ by $2 L_{1}$, with a Zero Average Exit $\operatorname{Velocity}\left(U_{0}=0\right)$}

\section{A. Analytical Results}

The solutions to this problem can be obtained from the previous solutions using the fact that free molecular flow out of a concentered rectangular exit is equivalent to a large rectangular source minus a smaller rectangular sink. The final number density result is:

$$
\begin{aligned}
n(X, Y, Z)= & \frac{1}{4 \pi}\left[\arctan \frac{(L-Y)(H-Z)}{X \sqrt{X^{2}+(L-Y)^{2}+(H-Z)^{2}}}\right. \\
& +\arctan \frac{(L-Y)(H+Z)}{X \sqrt{X^{2}+(L-Y)^{2}+(H+Z)^{2}}}+\arctan \frac{(L+Y)(H-Z)}{X \sqrt{X^{2}+(L+Y)^{2}+(H-Z)^{2}}} \\
& +\arctan \frac{(L+Y)(H+Z)}{X \sqrt{X^{2}+(L+Y)^{2}+(H+Z)^{2}}}-\arctan \frac{\left(L_{1}-Y\right)\left(H_{1}-Z\right)}{X \sqrt{X^{2}+\left(L_{1}-Y\right)^{2}+\left(H_{1}-Z\right)^{2}}} \\
& -\arctan \frac{\left(L_{1}-Y\right)\left(H_{1}+Z\right)}{X \sqrt{X^{2}+\left(L_{1}-Y\right)^{2}+\left(H_{1}+Z\right)^{2}}}-\arctan \frac{\left(L_{1}+Y\right)\left(H_{1}-Z\right)}{X \sqrt{X^{2}+\left(L_{1}+Y\right)^{2}+\left(H_{1}-Z\right)^{2}}} \\
& \left.-\arctan \frac{\left(L_{1}+Y\right)\left(H_{1}+Z\right)}{X \sqrt{X^{2}+\left(L_{1}+Y\right)^{2}+\left(H_{1}+Z\right)^{2}}}\right]
\end{aligned}
$$


The velocities along the $X, Y, Z$ directions are:

$$
\begin{aligned}
& \frac{U(X, Y, Z)}{\sqrt{2 R T_{0}}}=\frac{1}{4 n \pi \sqrt{\pi}}\left[\frac{H-Z}{\sqrt{(H-Z)^{2}+X^{2}}}\left(\arctan \frac{L-Y}{\sqrt{(H-Z)^{2}+X^{2}}}+\arctan \frac{L+Y}{\sqrt{(H-Z)^{2}+X^{2}}}\right)\right. \\
& +\frac{H+Z}{\sqrt{(H+Z)^{2}+X^{2}}}\left(\arctan \frac{L-Y}{\sqrt{(H+Z)^{2}+X^{2}}}+\arctan \frac{L+Y}{\sqrt{(H+Z)^{2}+X^{2}}}\right) \\
& +\frac{L-Y}{\sqrt{(L-Y)^{2}+X^{2}}}\left(\arctan \frac{H-Z}{\sqrt{(L-Y)^{2}+X^{2}}}+\arctan \frac{H+Z}{\sqrt{(L-Y)^{2}+X^{2}}}\right) \\
& +\frac{L+Y}{\sqrt{(L+Y)^{2}+X^{2}}}\left(\arctan \frac{H-Z}{\sqrt{(L+Y)^{2}+X^{2}}}+\arctan \frac{H+Z}{\sqrt{(L+Y)^{2}+X^{2}}}\right) \\
& -\frac{H_{1}-Z}{\sqrt{\left(H_{1}-Z\right)^{2}+X^{2}}}\left(\arctan \frac{L_{1}-Y}{\sqrt{\left(H_{1}-Z\right)^{2}+X^{2}}}+\arctan \frac{L_{1}+Y}{\sqrt{\left(H_{1}-Z\right)^{2}+X^{2}}}\right) \\
& -\frac{H_{1}+Z}{\sqrt{\left(H_{1}+Z\right)^{2}+X^{2}}}\left(\arctan \frac{L_{1}-Y}{\sqrt{\left(H_{1}+Z\right)^{2}+X^{2}}}+\arctan \frac{L_{1}+Y}{\sqrt{\left(H_{1}+Z\right)^{2}+X^{2}}}\right) \\
& -\frac{L_{1}-Y}{\sqrt{\left(L_{1}-Y\right)^{2}+X^{2}}}\left(\arctan \frac{H_{1}-Z}{\sqrt{\left(L_{1}-Y\right)^{2}+X^{2}}}+\arctan \frac{H_{1}+Z}{\sqrt{\left(L_{1}-Y\right)^{2}+X^{2}}}\right) \\
& \left.-\frac{L_{1}+Y}{\sqrt{\left(L_{1}+Y\right)^{2}+X^{2}}}\left(\arctan \frac{H_{1}-Z}{\sqrt{\left(L_{1}+Y\right)^{2}+X^{2}}}+\arctan \frac{H_{1}+Z}{\sqrt{\left(L_{1}+Y\right)^{2}+X^{2}}}\right)\right] \\
& \frac{V(X, Y, Z)}{\sqrt{2 R T_{0}}}=\frac{X}{4 n \pi \sqrt{\pi}}\left[\frac { 1 } { \sqrt { ( L - Y ) ^ { 2 } + X ^ { 2 } } } \left(\arctan \frac{H-Z}{\sqrt{(L-Y)^{2}+X^{2}}}\right.\right. \\
& \left.+\arctan \frac{H+Z}{\sqrt{(L-Y)^{2}+X^{2}}}\right)-\frac{1}{\sqrt{(L+Y)^{2}+X^{2}}}\left(\arctan \frac{H-Z}{\sqrt{(L+Y)^{2}+X^{2}}}\right. \\
& \left.+\arctan \frac{H+Z}{\sqrt{(L+Y)^{2}+X^{2}}}\right)-\frac{1}{\sqrt{\left(L_{1}-Y\right)^{2}+X^{2}}}\left(\arctan \frac{H_{1}-Z}{\sqrt{\left(L_{1}-Y\right)^{2}+X^{2}}}\right. \\
& \left.\left.+\arctan \frac{H_{1}+Z}{\sqrt{\left(L_{1}-Y\right)^{2}+X^{2}}}\right)+\frac{1}{\sqrt{\left(L_{1}+Y\right)^{2}+X^{2}}}\left(\arctan \frac{H_{1}-Z}{\sqrt{\left(L_{1}+Y\right)^{2}+X^{2}}}+\arctan \frac{H_{1}+Z}{\sqrt{\left(L_{1}+Y\right)^{2}+X^{2}}}\right)\right] \\
& \frac{W(X, Y, Z)}{\sqrt{2 R T_{0}}}=\frac{X}{4 n \pi \sqrt{\pi}}\left[\frac{1}{\sqrt{(H-Z)^{2}+X^{2}}}\left(\arctan \frac{L-Y}{\sqrt{(H-Z)^{2}+X^{2}}}+\arctan \frac{L+Y}{\sqrt{(H-Z)^{2}+X^{2}}}\right)\right. \\
& -\frac{1}{\sqrt{(H+Z)^{2}+X^{2}}}\left(\arctan \frac{L-Y}{\sqrt{(H+Z)^{2}+X^{2}}}+\arctan \frac{L+Y}{\sqrt{(H+Z)^{2}+X^{2}}}\right) \\
& -\frac{1}{\sqrt{\left(H_{1}-Z\right)^{2}+X^{2}}}\left(\arctan \frac{L_{1}-Y}{\sqrt{\left(H_{1}-Z\right)^{2}+X^{2}}}+\arctan \frac{L_{1}+Y}{\sqrt{\left(H_{1}-Z\right)^{2}+X^{2}}}\right) \\
& \left.+\frac{1}{\sqrt{\left(H_{1}+Z\right)^{2}+X^{2}}}\left(\arctan \frac{L_{1}-Y}{\sqrt{\left(H_{1}+Z\right)^{2}+X^{2}}}+\arctan \frac{L_{1}+Y}{\sqrt{\left(H_{1}+Z\right)^{2}+X^{2}}}\right)\right]
\end{aligned}
$$

Along the exit centerline where $Y=Z=0$ :

$$
\begin{aligned}
n(X, 0,0)=\frac{1}{\pi} & {\left[\arctan \frac{H L}{X \sqrt{X^{2}+H^{2}+L^{2}}}-\arctan \frac{H_{1} L_{1}}{X \sqrt{X^{2}+H_{1}^{2}+L_{1}^{2}}}\right] } \\
\frac{U(X, 0,0)}{\sqrt{2 R T_{0}}=} & \frac{1}{n \pi \sqrt{\pi}}\left[\frac{H}{\sqrt{H^{2}+X^{2}}} \arctan \frac{L}{\sqrt{X^{2}+H^{2}}}+\frac{L}{\sqrt{X^{2}+L^{2}}} \arctan \frac{H}{\sqrt{X^{2}+L^{2}}}\right. \\
& \left.-\frac{H_{1}}{\sqrt{H_{1}^{2}+X^{2}}} \arctan \frac{L_{1}}{\sqrt{X^{2}+H_{1}^{2}}}-\frac{L_{1}}{\sqrt{X^{2}+L_{1}^{2}}} \arctan \frac{H_{1}}{\sqrt{X^{2}+L_{1}^{2}}}\right]
\end{aligned}
$$

These centerline results contain $H, L, H_{1}$ and $L_{1}$, and they are quite different from the standard results of free molecular flow through a small circular orifice. When $X$ changes from 0 to $\infty, n$ changes from $\frac{1}{2}$ to 0 , while $U(0,0,0)=\frac{1}{\sqrt{\pi}}$ with $H=L$ and $H_{1}=L_{1}$.

\section{B. DSMC Simulations and Discussions}

A three-dimensional DSMC simulation is performed to simulate the effusion flow from a concentered rectangular exit with $2 L=2 \times 0.2 \mathrm{~m}$ by $2 H=2 \times 0.2 \mathrm{~m}$ and $2 L_{1}=2 \times 0.05 \mathrm{~m}$ by $2 H_{1}=2 \times 0.05 \mathrm{~m}$. Due to the symmetry in the geometry configuration, only a quarter of the exit is included in the simulation domain.

13 of 33

American Institute of Aeronautics and Astronautics 
The simulation domain is a cubic shape with side length of 1 meter. A tetrahedral mesh of 2 million cells is used to represent the whole domain, and in the final sampling state, about 6 million particles are maintained. As usual, the collision function in MONACO was turned off.

Figure 10 shows the number density contours in the plane $\mathrm{Y}=0$, the solid contours are the analytical results obtained in this study while the dashed contours are the DSMC results. At the exit, both the analytical and DSMC results yield the same value of $\frac{1}{2}$. Figures 11 and 12 show contours of $U(X, 0, Z)$ and $W(X, 0, Z)$ in the plane $\mathrm{Y}=0$. In general, both comparisons are quite satisfactory. In all of these results, the exit effects are well captured. The contours of number density and velocities in the plane $Z=0$ have similar patterns so they are omitted here.

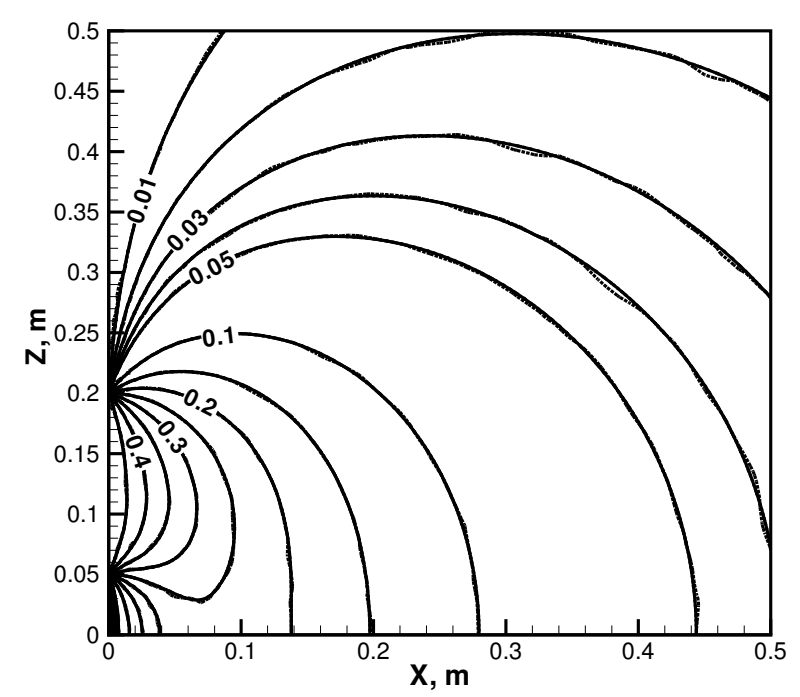

Figure 10. Case 3: Contours of Number Density $\left(H=L=0.2 m, H_{1}=L_{1}=0.05 m, U_{0}=\mathbf{0}\right.$, Solid: Analytical, Dashed: DSMC without Collisions).

Figure 13 shows profiles of number density along one horizontal line passing the exit corners $(Y=L$, $Z=H$ and $Y=L_{1}, Z=H_{1}$ ) and the exit centerline. In general, the number density distribution fits quite accurately, and at the outer exit corner the density is about $\frac{1}{8}$ while at the inner corner the value is $\frac{3}{8}$. This is physically correct, because the inner exit corner has a 3 times larger neighborhood than the outer exit corner point. It is possible to further obtain some specific properties, such as the location on the axis with the highest number density, but these derivation processes involve finding roots of higher order equations, and they are omitted in this paper.

\section{Problem Four: Free Molecular Effusion Flow Out of a Circular Exit with a Zero Average Speed $\left(U_{0}=0\right)$}

\section{A. Analytical Results}

For this case, the velocity-position relation takes a new format. Suppose the average velocity at the circular exit is zero. From any point $(0, y, z)$ on the exit which is characterized by a radius of $R$, only particles with the following special velocity components can arrive at a point $(X, 0, Z)$ in front of the exit:

$$
\frac{X}{u}=\frac{Y-y}{v}=\frac{Z-z}{w}
$$

14 of 33 


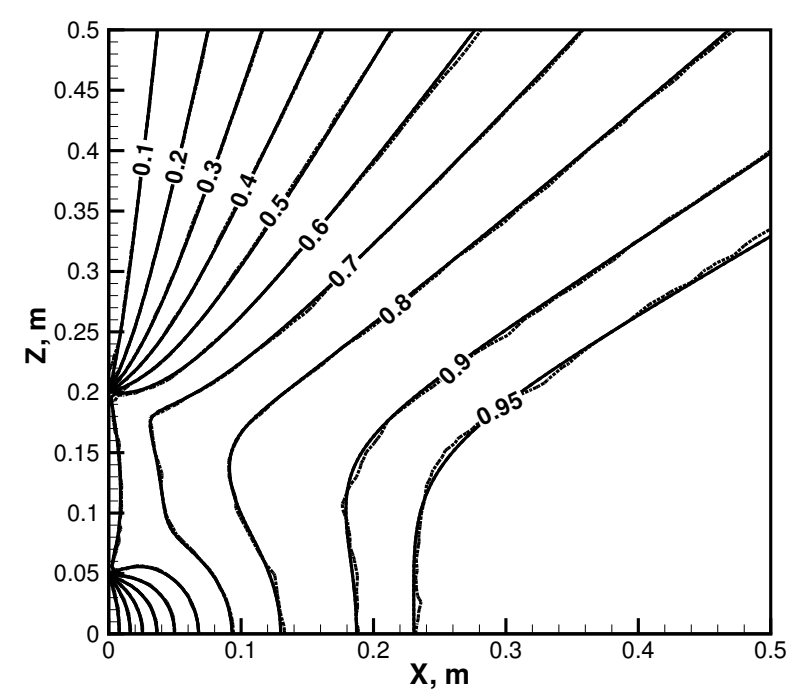

Figure 11. Case 3: Contours of $\frac{U(X, 0, Z)}{\sqrt{2 R T_{0}}}\left(H=L=0.2 m, H_{1}=L_{1}=0.05 m, U_{0}=\mathbf{0}\right.$, Solid: Analytical, Dashed: DSMC without Collisions).

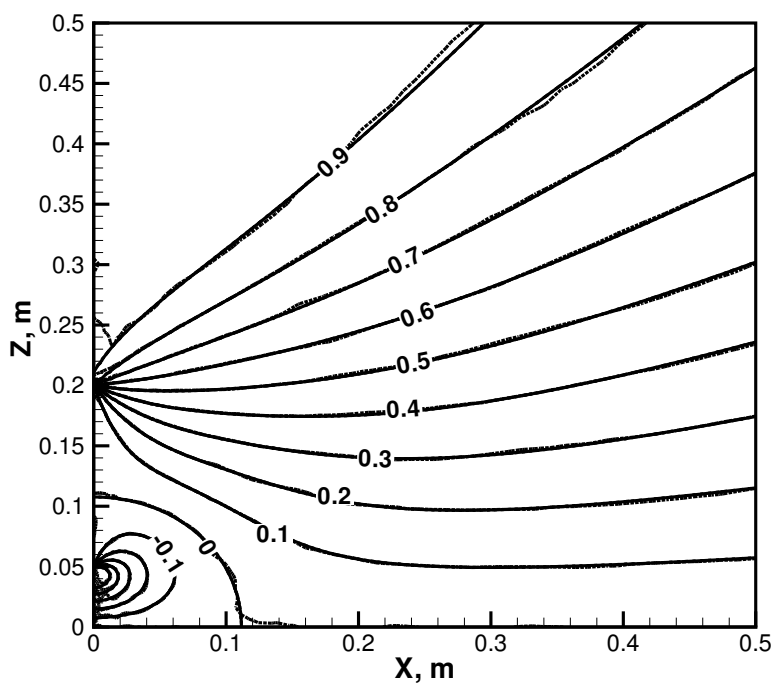

Figure 12. Case 3: Contours of $\frac{W(X, 0, Z)}{\sqrt{2 R T_{0}}}\left(H=L=0.2 m, H_{1}=L_{1}=0.05 m, U_{0}=\mathbf{0}\right.$, Solid: Analytical, Dashed: DSMC without Collisions).

15 of 33

American Institute of Aeronautics and Astronautics 


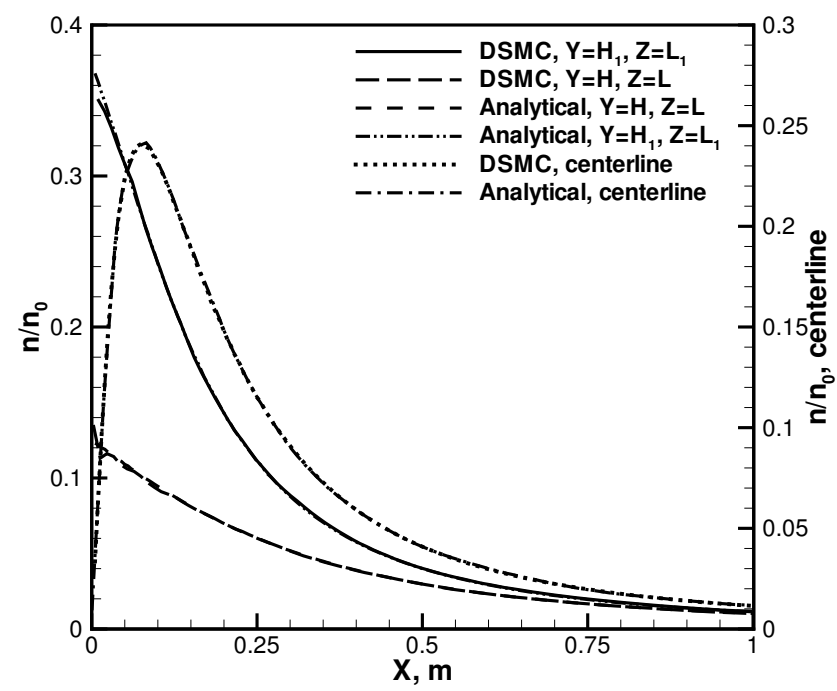

Figure 13. Case 3: Profiles of $n / n_{0}\left(H=L=0.2 m, H_{1}=L_{1}=0.05 m, U_{0}=\mathbf{0}\right)$.

where $X>0, Y>0, Z>0$. Combined with geometry relations:

$$
z=r \sin \theta=Z-X w / u, y=r \cos \theta=Y-X v / u
$$

where $r \in[0, R], \theta \in[0,2 \pi]$, the integrals for the number density and the velocities can be simplified using the following variable change:

$$
d v d w=\left|\begin{array}{ll}
\frac{\partial v}{\partial r} & \frac{\partial v}{\partial \theta} \\
\frac{\partial w}{\partial r} & \frac{\partial w}{\partial \theta}
\end{array}\right| d r d \theta=\frac{u^{2}}{X^{2}} r d r d \theta
$$

The final results for number density at a point $(X, 0, Z)$ in front of the exit contains an integral term: ${ }^{14}$

$$
n(X, 0, Z)=\frac{1}{2}-\frac{X}{4 \pi} \int_{-\pi}^{\pi}\left[\frac{\left(X^{2}+Z^{2}-Z R \sin \theta\right) d \theta}{\left(X^{2}+Z^{2} \cos ^{2} \theta\right) \sqrt{X^{2}+Z^{2}+R^{2}-2 R Z \sin \theta}}\right]
$$

An option to further simplify the above expression is to expand with a series of trigonometric functions. However, this treatment does not save much evaluation cost, and to maintain higher accuracy, this format is kept and a numerical evaluation is performed.

Similarly, the velocity along the axial direction is:

$$
\begin{aligned}
\frac{U(X, 0, Z)}{\sqrt{2 R T_{0}}}= & \frac{X^{2}}{2 \pi n \sqrt{\pi}}\left(\frac{\pi}{X \sqrt{X^{2}+Z^{2}}}-\frac{\pi}{\sqrt{\left(X^{2}+Z^{2}+R^{2}\right)^{2}-4 R^{2} Z^{2}}}+\int_{-\pi}^{\pi}[\right. \\
& \frac{Z \sin \theta}{2\left(X^{2}+Z^{2} \cos ^{2} \theta\right)^{3 / 2}} \arctan \frac{Z \sin \theta}{\sqrt{X^{2}+Z^{2} \cos ^{2} \theta}}+\frac{Z \sin \theta(R-Z \sin \theta) d \theta}{2\left(X^{2}+Z^{2}+R^{2}-2 R Z \sin \theta\right)\left(X^{2}+Z^{2} \cos ^{2} \theta\right)} \\
& \left.\left.+\frac{Z \sin \theta d \theta}{2\left(X^{2}+Z^{2} \cos ^{2} \theta\right)^{3 / 2}} \arctan \frac{R-Z \sin \theta}{\sqrt{X^{2}+Z^{2} \cos ^{2} \theta}}\right] d \theta\right)
\end{aligned}
$$

16 of 33 


$$
\begin{aligned}
\frac{W(X, 0, Z)}{\sqrt{2 R T_{0}}}= & \frac{1}{2 \pi n \sqrt{\pi}} \int_{0}^{2 \pi}\left(\frac{-(Z / 2) \cos (2 \theta) d \theta}{\left[X^{2}+R^{2}+Z^{2}-2 R Z \sin \theta\right]}-\frac{\sin (\theta) d \theta}{\sqrt{X^{2}+Z^{2} \cos ^{2} \theta}}\left(\arctan \frac{R-Z \sin \theta}{\sqrt{X^{2}+Z^{2} \cos ^{2} \theta}}\right.\right. \\
& \left.+\arctan \frac{Z \sin \theta}{\sqrt{X^{2}+Z^{2} \cos ^{2} \theta}}\right)++\frac{\sin \theta\left(X^{2}+2 Z^{2} \cos ^{2} \theta\right) d \theta}{2\left(X^{2}+Z^{2} \cos ^{2} \theta\right)^{3 / 2}}\left(\arctan \frac{R-Z \sin \theta}{\sqrt{X^{2}+Z^{2} \cos ^{2} \theta}}+\arctan \frac{Z \sin \theta}{\sqrt{X^{2}+Z^{2} \cos ^{2} \theta}}\right) \\
& \left.+\frac{\sin \theta\left(X^{2}+2 Z^{2} \cos ^{2} \theta\right)(R-Z \sin \theta) d \theta}{2\left(X^{2}+Z^{2} \cos ^{2} \theta\right)\left(X^{2}+Z^{2}+R^{2}-2 R Z \sin \theta\right)}+\frac{Z \sin ^{2} \theta\left(X^{2}+2 Z^{2} \cos ^{2} \theta\right) d \theta}{2\left(X^{2}+Z^{2} \cos ^{2} \theta\right)\left(X^{2}+Z^{2}\right)}\right)
\end{aligned}
$$

Along the $\mathrm{X}$-axis passing through the exit center, the number density and velocity variations are:

$$
n(X, 0,0)=\frac{1}{2}-\frac{X}{2 \sqrt{X^{2}+R^{2}}}, \frac{U(X, 0,0)}{\sqrt{2 R T_{0}}}=\frac{1}{\sqrt{\pi}}\left(1+\frac{X}{\sqrt{X^{2}+R^{2}}}\right)
$$

when $X$ changes from 0 to $\infty, n$ changes from $\frac{1}{2}$ to 0 , while $\frac{U(X, 0,0)}{\sqrt{2 R T_{0}}}$ changes from $\frac{1}{\sqrt{\pi}}$ to $\frac{2}{\sqrt{\pi}}$.

\section{B. Simulations and Discussions}

An axi-symmetric DSMC simulation is performed to validate the analytical results obtained in this case. The simulation domain size is $0.8 \mathrm{~m}$ by $0.8 \mathrm{~m}$, and a rectangular mesh of 28,800 cells is adopted mainly to provide high resolution. The circular exit diameter is set to $0.1 \mathrm{~m}$ and the number density at the exit is set to a value resulting in a Knudsen number of 20 based on the characteristic length of the exit diameter. In the final sampling stage, about 2 million particles are maintained in the simulation domain. By turning off the collision function in MONACO, the simulation results are expected to be close to these analytical results. The gas translational temperature at the exit is set to $300 \mathrm{~K}$.

Figures 14 and 15 show the analytical results of number density and $\frac{U(X, 0, Z)}{\sqrt{2 R T_{0}}}$ at plane $Z=0$. The flow patterns are quite similar to Case 1 , effusion flow from a thin slit. Figure 16 shows a comparison of $\frac{W(X, 0, Z)}{\sqrt{2 R T_{0}}}$. The comparison shows exactly the same flow patterns, and almost identical results. These velocity results indicate clearly the finite geometry exit has an influence on the flowfield around the exit.

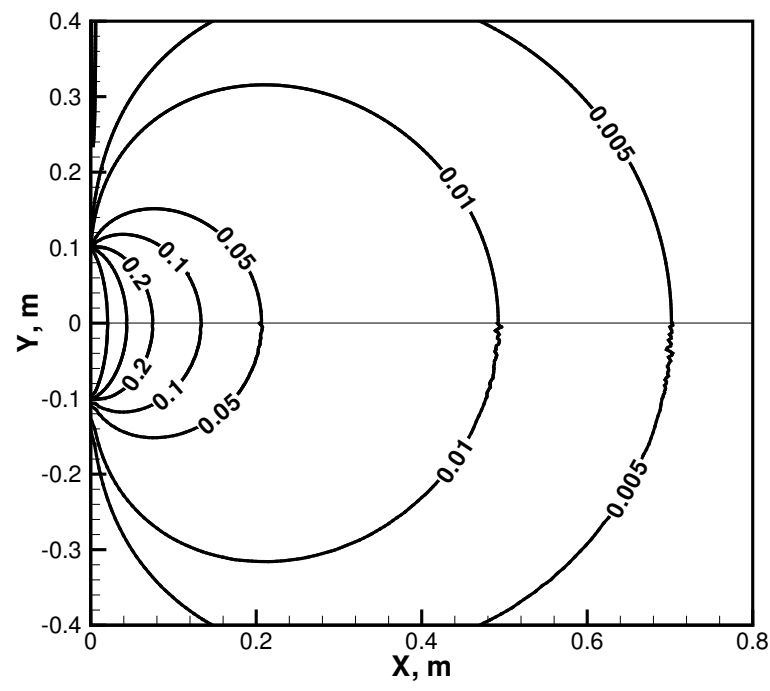

Figure 14. Case 4: Contours of Number Density $\left(R=0.1 \mathrm{~m}, U_{0}=0\right.$, Top: Analytical, Bottom: DSMC without Collisions).

17 of 33 
Figures 17 and 18 show variations of number density and velocity along two horizontal lines, one passing through the exit center and the other passing through the edge of this exit. It is interesting to recognize that the number density at $(0, R)$ is close to $\frac{1}{4}$, almost half of the value at the exit center.

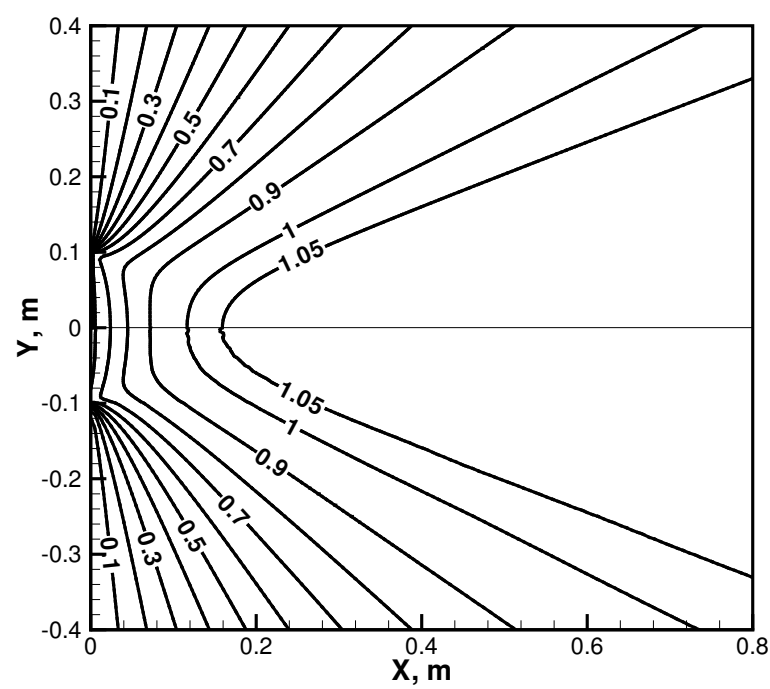

Figure 15. Case 4: Speed Ratio $\frac{U(X, Y, 0)}{\sqrt{2 R T_{0}}}\left(R=0.1 \mathrm{~m}, U_{0}=\mathbf{0}\right.$, Top: Analytical, Bottom: DSMC).

\section{Problem Five: Free Molecular Effusion Flow Out of an Annular Exit with a Zero Average Exit Velocity $\left(U_{0}=0\right)$}

\section{A. Analytical Results}

An annulus is a common shape for the exit of the acceleration channel of Hall thrusters. Though this shape is characterized by two parameters of inner and outer radius of $R_{1}$ and $R_{2}$, Equations 36- 38 are still correct for this situation. The only difference in the derivation process is the integral domain $r \in\left[R_{1}, R_{2}\right]$ for an annular exit instead of $r \in[0, R]$ for a circular exit. Hence, by observing the integral domains, the free molecular flow out of an annulus can be considered as a large circular source of $R_{2}$ minus a small circular sink of $R_{1}$.

The final results of number density and velocities at a point $(X, 0, Z)$ are:

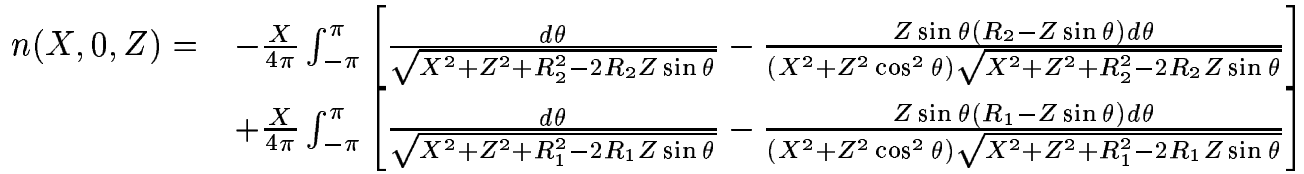

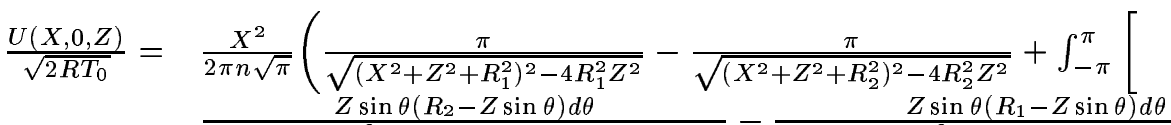

$$
\begin{aligned}
& \frac{2\left(X^{2}+Z^{2}+R_{2}^{2}-2 R_{2} Z \sin \theta\right)\left(X^{2}+Z^{2} \cos ^{2} \theta\right)}{2\left(X^{2}+Z^{2}+R_{1}^{2}-2 R_{1} Z \sin \theta\right)\left(X^{2}+Z^{2} \cos ^{2} \theta\right)} \\
& \left.\left.+\frac{Z \sin \theta d \theta}{2\left(X^{2}+Z^{2} \cos ^{2} \theta\right)^{3 / 2}} \arctan \frac{R_{2}-Z \sin \theta}{\sqrt{X^{2}+Z^{2} \cos ^{2} \theta}}-\frac{Z \sin \theta d \theta}{2\left(X^{2}+Z^{2} \cos ^{2} \theta\right)^{3 / 2}} \arctan \frac{R_{1}-Z \sin \theta}{\sqrt{X^{2}+Z^{2} \cos ^{2} \theta}}\right] d \theta\right)
\end{aligned}
$$

18 of 33

American Institute of Aeronautics and Astronautics 


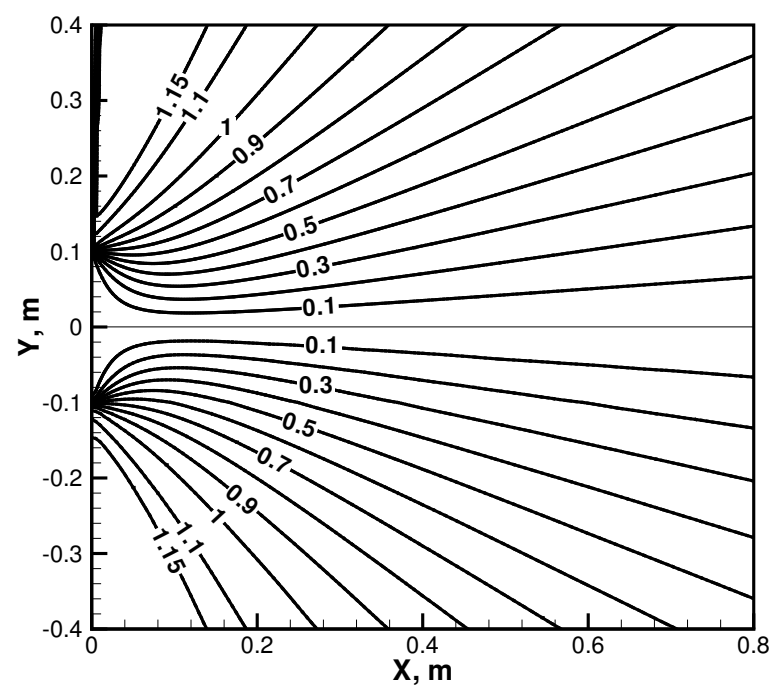

Figure 16. Case 4: Speed Ratio $\frac{W(X, Y, 0)}{\sqrt{2 R T_{0}}}\left(R=\mathbf{0 . 1} \mathbf{~ m}, U_{0}=\mathbf{0}\right.$, Top: Analytical, Bottom: DSMC).

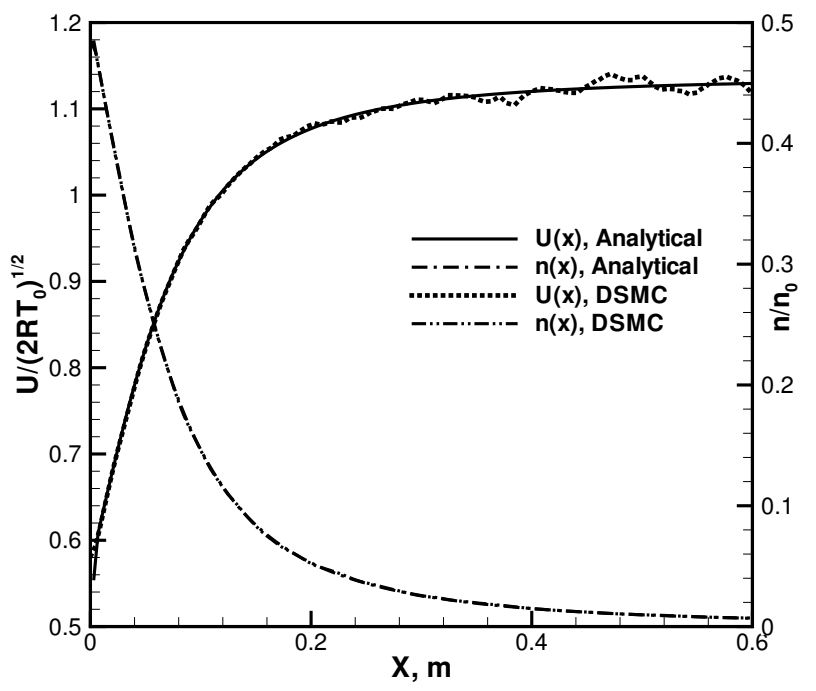

Figure 17. Case 4: Normalized Number Density and Velocity along Centerline $\left(R=0.1 \mathrm{~m}, U_{0}=0\right)$.

19 of 33

American Institute of Aeronautics and Astronautics 


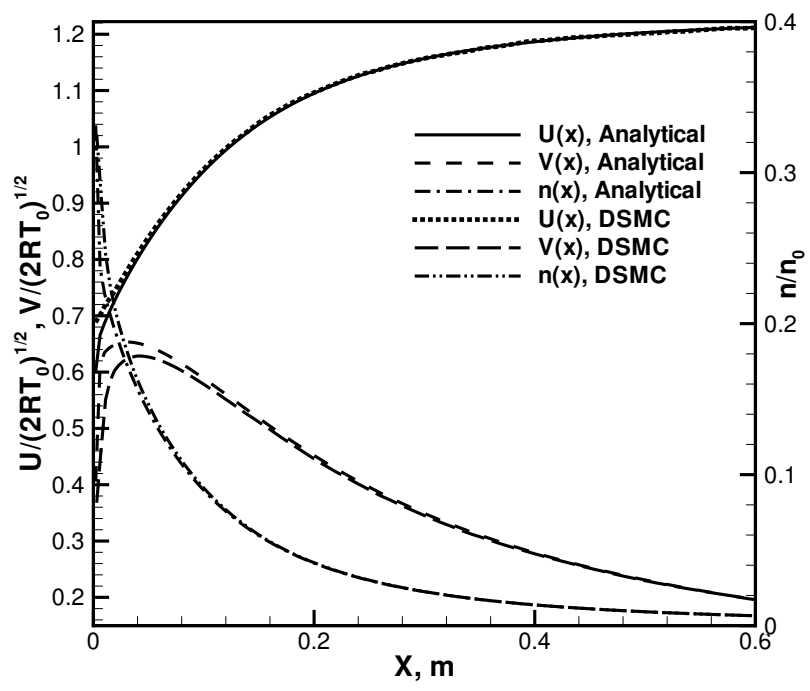

Figure 18. Case 4: Normalized Number Density and Velocity along Exit Tip $\left(R=0.1 \mathrm{~m}, U_{0}=0\right)$.

$$
\begin{aligned}
\frac{W(X, 0, Z)}{\sqrt{2 R T_{0}}}= & \frac{1}{2 \pi n \sqrt{\pi}} \int_{0}^{2 \pi}\left[\frac{-(Z / 2) \cos (2 \theta) d \theta}{\left[X^{2}+R_{2}^{2}+Z^{2}-2 R_{2} Z \sin \theta\right]}+\frac{(Z / 2) \cos (2 \theta) d \theta}{\left[X^{2}+R_{1}^{2}+Z^{2}-2 R_{1} Z \sin \theta\right]}\right. \\
& -\frac{\sin (\theta) d \theta}{\sqrt{X^{2}+Z^{2} \cos ^{2} \theta}}\left(\arctan \frac{R_{2}-Z \sin \theta}{\sqrt{X^{2}+Z^{2} \cos ^{2} \theta}}-\arctan \frac{R_{1}-Z \sin \theta}{\sqrt{X^{2}+Z^{2} \cos ^{2} \theta}}\right) \\
& +\frac{\sin \theta\left(X^{2}+2 Z^{2} \cos ^{2} \theta\right) d \theta}{2\left(X^{2}+Z^{2} \cos ^{2} \theta\right)^{3 / 2}}\left(\arctan \frac{R_{2}-Z \sin \theta}{\sqrt{X^{2}+Z^{2} \cos ^{2} \theta}}-\arctan \frac{R_{1}-Z \sin \theta}{\sqrt{X^{2}+Z^{2} \cos ^{2} \theta}}\right) \\
& \left.+\frac{\sin \theta\left(X^{2}+2 Z^{2} \cos ^{2} \theta\right)\left(R_{2}-Z \sin \theta\right) d \theta}{2\left(X^{2}+Z^{2} \cos ^{2} \theta\right)\left(X^{2}+Z^{2}+R_{2}^{2}-2 R_{2} Z \sin \theta\right)}-\frac{\sin \theta\left(X^{2}+2 Z^{2} \cos ^{2} \theta\right)\left(R_{1}-Z \sin \theta\right) d \theta}{2\left(X^{2}+Z^{2} \cos ^{2} \theta\right)\left(X^{2}+Z^{2}+R_{1}^{2}-2 R_{1} Z \sin \theta\right)}\right]
\end{aligned}
$$

From the above relation, the centerline variations are:

$$
n(X, 0,0)=\frac{X}{2 \sqrt{X^{2}+R_{1}^{2}}}-\frac{X}{2 \sqrt{X^{2}+R_{2}^{2}}}, \frac{U(X, 0,0)}{\sqrt{2 R T_{0}}}=\frac{X}{\sqrt{\pi}}\left(\frac{1}{\sqrt{X^{2}+R_{1}^{2}}}+\frac{1}{\sqrt{X^{2}+R_{2}^{2}}}\right)
$$

Obviously, when $X$ changes from 0 to $\infty$, the centerline number density increases from 0 to a specific value, then decreases slowly to zero, while the speed ratio $\frac{U(X, 0,0)}{\sqrt{2 R T_{0}}}$ increases from 0 to $\frac{1}{2 \sqrt{\pi}}$.

\section{B. Simulations and Discussions}

An axi-symmetric DSMC simulation without collisions is performed to validate these analytical results. The inner and outer radii of the annulus are set to $R_{1}=0.2 \mathrm{~m}$ and $R_{2}=0.4 \mathrm{~m}$, respectively. About 19,200 rectangular cells are used to represent a square simulation domain of $1.2 \mathrm{~m}$ by $1.2 \mathrm{~m}$. As usual, the temperature at the exit is set to $300 \mathrm{~K}$ while the number density is set to a value achieving a Knudsen number of 20 based on the characteristic length of $R_{2}-R_{1}$. At the final sampling stage, about 1.2 million particles are maintained.

Figure 19 shows contours of number density, the bottom contours are the analytical results and the top contours represent the DSMC results. In the whole simulation domain, the comparison shows almost identical results. Figures 20 and 21 show the contours of velocities. Both comparisons are quite satisfactory as well. In all of these three results, both the exit region and the slow "cavity" region in the center, which

20 of 33

American Institute of Aeronautics and Astronautics 


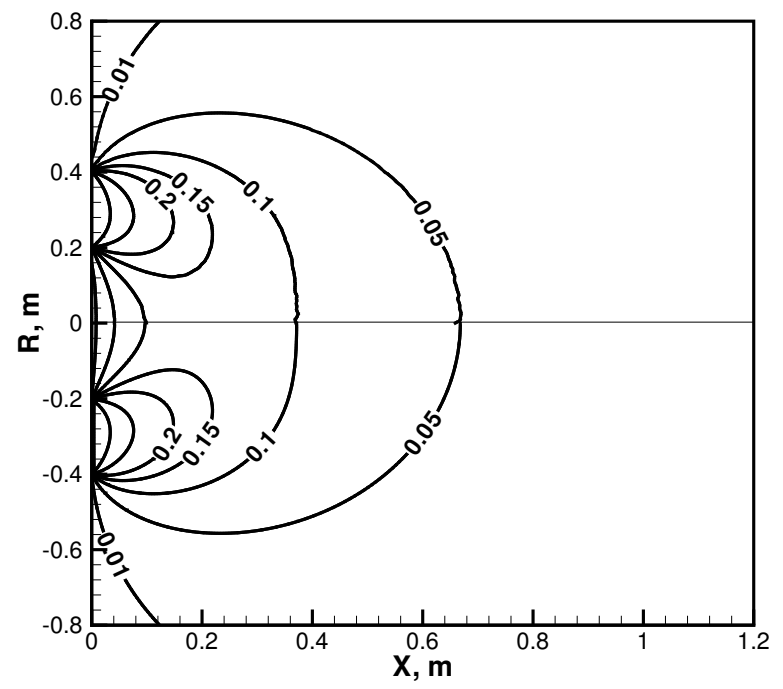

Figure 19. Case 4: Contours of Normalized Number Density $\left(R_{1}=0.2 \mathrm{~m}, R_{2}=0.4 \mathrm{~m}, U_{0}=0\right.$, Bottom: Analytical, Top: DSMC).

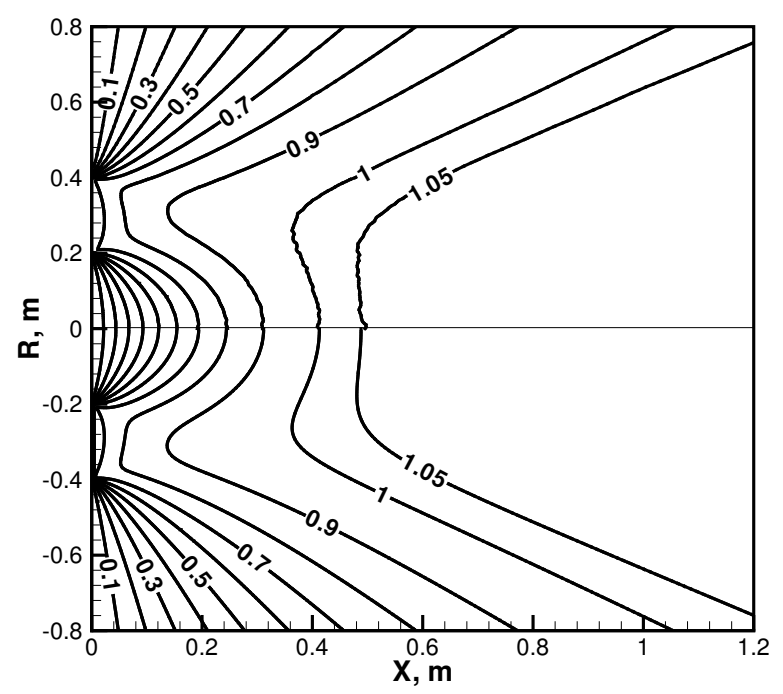

Figure 20. Case 4: Contours of $\frac{U(X, 0, R)}{\sqrt{2 R T_{0}}}\left(R_{1}=0.2 \mathrm{~m}, R_{2}=0.4 \mathrm{~m}, U_{0}=0\right.$, Bottom: Analytical, Top: DSMC). 


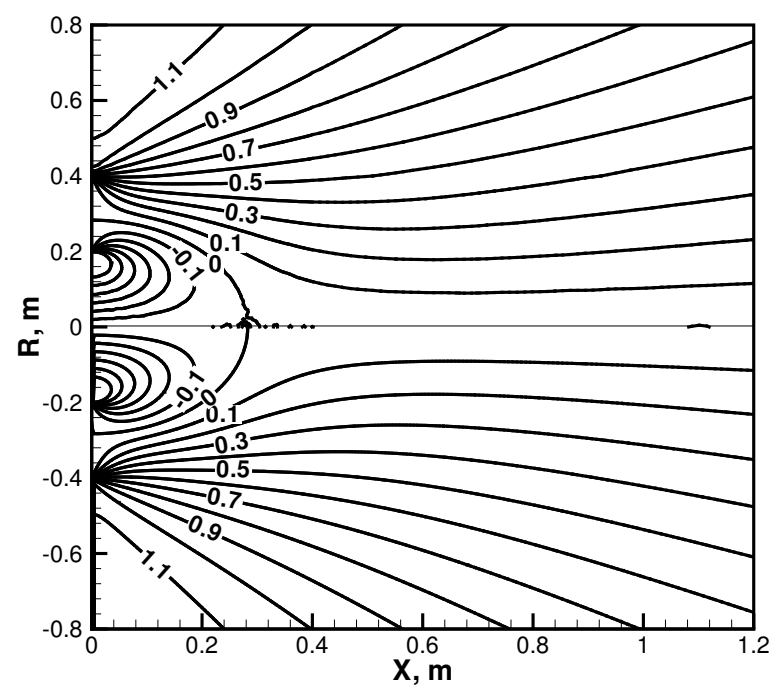

Figure 21. Case 4: Contours of $\frac{W(X, 0, R)}{\sqrt{2 R T_{0}}}\left(R_{1}=0.2 \mathrm{~m}, R_{2}=\mathbf{0 . 4} \mathbf{m}, U_{0}=\mathbf{0}\right.$, Bottom: Analytical, Top: DSMC).

is characterized by negative $W_{z}$, are clearly captured. There are two branches of zero-value lines for $W_{z}$, one is along the $\mathrm{X}$-axis, while the other curved branch starts from a point inside the exit and ends on the $\mathrm{X}$-axis. The intersection point of the two branches can be found analytically from Equations 45 . On the specific point,

$$
\left.\frac{\partial W(X, 0, Z)}{\partial Z}\right|_{Z=0}=0
$$

From this condition, it is straightforward to show that $X=\sqrt{R_{1} R_{2}}$ is the intersection point on the axis.

Figures 22, 23, 24 and 25 are number density and velocity variations along four horizontal lines with different radius to the axis: $r=R_{2}, r=\frac{1}{2}\left(R_{2}+R_{1}\right), r=R_{1}$ and $r=0$. It is quite evident that the number density at the exit tips are close to $\frac{1}{4}$ while on the exit center point the value is $\frac{1}{2}$. Generally, theoretical velocities and number densities match the results from numerical simulation.

One important result can be obtained for the location on the axis where the highest number density occurs. From Equation 43:

$$
\frac{\partial n(X, 0,0)}{\partial X}=\frac{\partial}{\partial X}\left(\frac{X}{2 \sqrt{X^{2}+R_{1}^{2}}}-\frac{X}{2 \sqrt{X^{2}+R_{2}^{2}}}\right)=0
$$

This leads to:

$$
\begin{gathered}
X_{*}=\sqrt{\frac{R_{2}^{2} R_{1}^{4 / 3}-R_{1}^{2} R_{2}^{4 / 3}}{R_{2}^{4 / 3}-R_{1}^{4 / 3}}}=\frac{R_{1}^{2 / 3} R_{2}^{2 / 3}}{\sqrt{R_{1}^{2 / 3}+R_{2}^{2 / 3}}}=\frac{\kappa^{2 / 3} R_{1}}{\sqrt{1+\kappa^{2 / 3}}} \\
n_{\max }=\frac{1}{2}\left(\kappa^{2 / 3}-1\right)\left(1+\kappa^{2 / 3}+\kappa^{4 / 3}\right)^{-1 / 2}
\end{gathered}
$$

where $\kappa=R_{2} / R_{1}>1$. Further notice that Equation 49 is an increasing function of $\kappa$, then a range of $X_{*}$ can be determined as:

$$
R_{1} / \sqrt{2}<X_{*}<\kappa^{1 / 3} R_{1}=\left(R_{1}^{2} R_{2}\right)^{1 / 3}<\frac{2 R_{1}+R_{2}}{3}
$$

\section{2 of 33}


It is interesting to notice that this distance is less than the average value of the inner and outer radius.

Equations 49-50 contain only the inner radius and outer radius of the annulus. This is because the derivation is based on a free molecular flow assumption without collision effects, hence the final expression contains only geometry factors, which is quite expected and reasonable. For an annulus with $R_{1}=0.2 \mathrm{~m}$ and $R_{2}=0.4 \mathrm{~m}$, the highest number density along the centerline is 0.1299 at $X=0.196 \mathrm{~m}$, which is shown in Figure 25. The DSMC simulation without collisions predicts the same value at the same location.

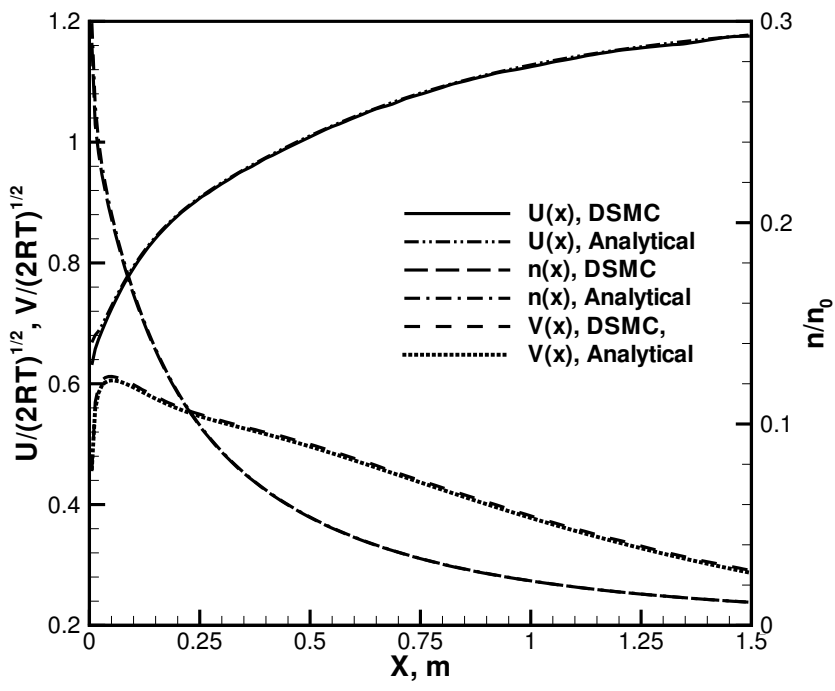

Figure 22. Case 5: Number Density and Normalized Velocities along $r=R_{2}\left(R_{1}=0.2 \mathrm{~m}, R_{2}=0.4 \mathrm{~m}, U_{0}=0\right)$.

\section{Problem Six: Free Molecular Flow Out of a Thin Slit with a Non-zero Average Speed $\left(U_{0}>0\right)$}

\section{A. Analytical Results}

Narasimha discussed the jet flow out of a point source with a non-zero average exit velocity. ${ }^{6}$ In this section, the finite width of the slit, $H$, is considered. Consider the velocity space for a point $(X, Y)$ in front of the slit. The velocity distribution at the point still follows Equation 2, but the integral domain changes significantly because of the non-zero average exit velocity. With this change, from any point $(0, h)$ on the slit, particles can arrive at the point $(X, Y)$ if and only if their velocity components satisfy the following relation:

$$
\tan (\theta)=\frac{Y-h}{X}=\frac{v}{u+U_{0}},-H<y<H .
$$

To compare the difference in the integral domains, for this case, the velocity distribution function does not shift to the right with a value of $U_{0}$. Hence, $u+U_{0}$ represents a particle's actual velocity along the $\mathrm{X}$-direction. Obviously the non-zero average velocity $U_{0}$ does not destroy the one-to-one mapping relation between velocity phase spaces.

The two integral domain boundaries are:

$$
\tan \left(\theta_{2}\right)=\frac{Y+H}{X}, \tan \left(\theta_{1}\right)=\frac{Y-H}{X}
$$

23 of 33 


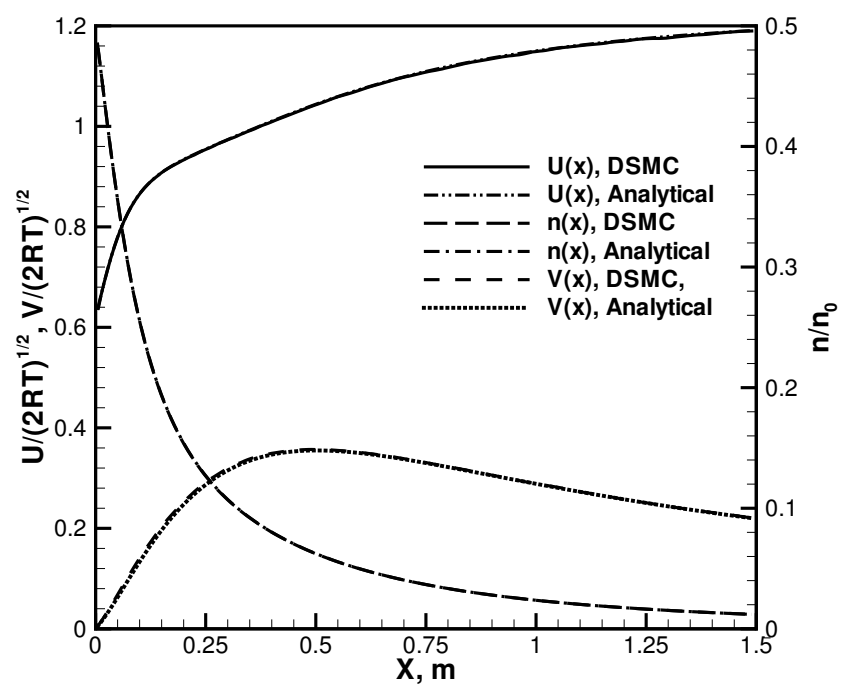

Figure 23. Case 5: Number Density and Normalized Velocities along $r=\frac{R_{2}+R_{1}}{2}\left(R_{1}=0.2 \mathrm{~m}, R_{2}=0.4 \mathrm{~m}, U_{0}=0\right)$.

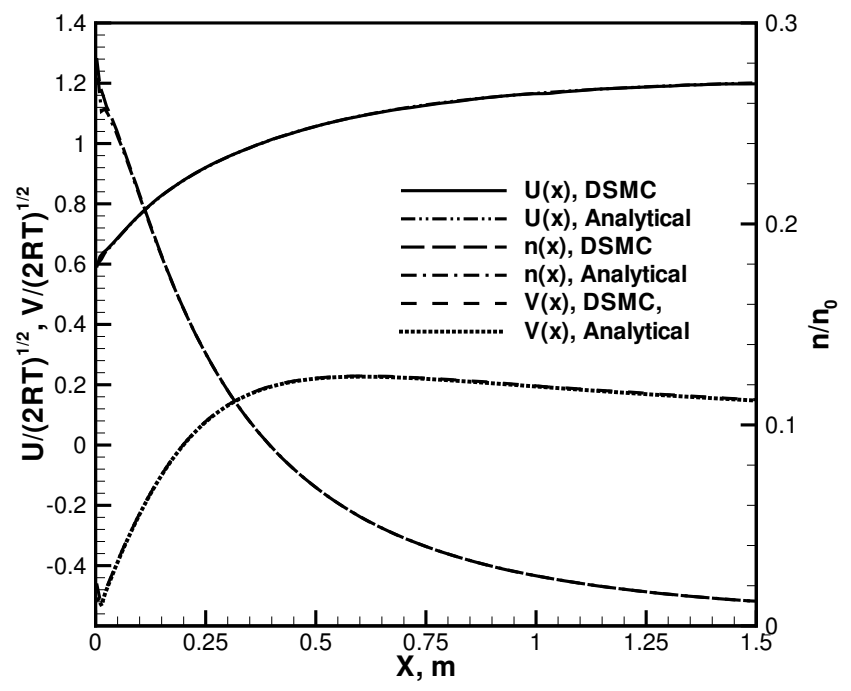

Figure 24. Case 5: Number Density and Normalized Velocities along $r=R_{1}\left(R_{1}=0.2 \mathrm{~m}, R_{2}=0.4 \mathrm{~m}, U_{0}=0\right)$. 


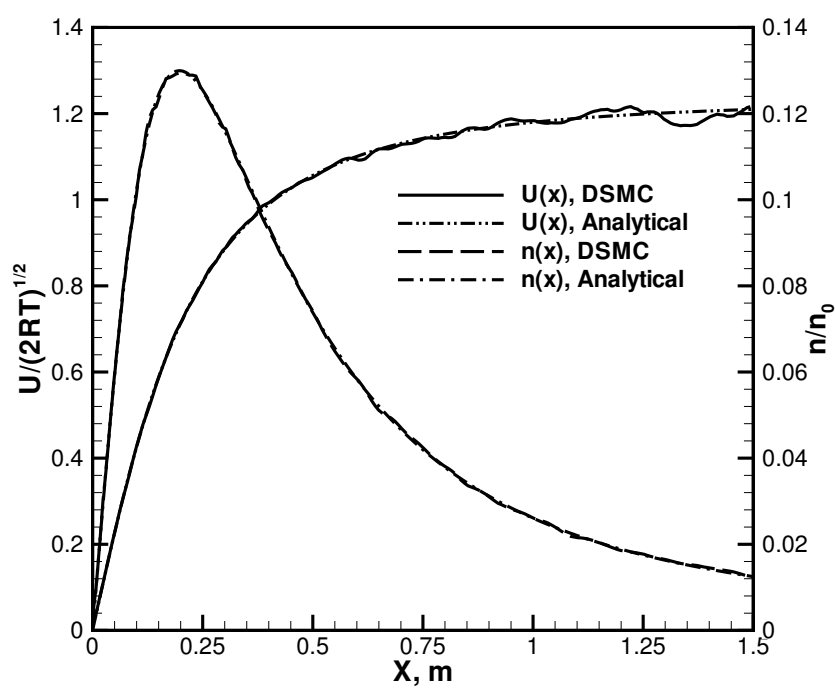

Figure 25. Case 5: Number Density and Normalized Velocities along $r=0\left(R_{1}=0.2 \mathrm{~m}, R_{2}=0.4 \mathrm{~m}, U_{0}=0\right)$.

Figure 26 shows the effect of the non-zero exit velocity on the integral domain in velocity space. On the left side is a case of zero average exit velocity and the right side is a case of non-zero average exit velocity. In both plots, region "Aoa" represents an integral domain for a point $(X, Y)$ out of the plume core $(Y>H)$, and region "Bob" represents an integral domain for a point in the plume core $(Y<H)$. The integral domain widens as the point $(X, Y)$ becomes closer to the slit. It is immediately observed that there are several changes between these two integral domains: The existence of a nonzero $U_{0}$ translates the integral domain to the left, without any effects on the slopes of the two domain boundaries. The two boundaries shift up or down, resulting in different effects on the points inside the plume core $(Y<H)$ and outside the plume core $(Y>H)$ : if the point is inside the core, the number density increases for the case of a non-zero average exit velocity because of a larger integral domain including regions close to the origin with the highest probability; while the number density at a point outside the plume core decreases because the origin point is excluded from the integral domain. Physically this is reasonable: with a higher average exit velocity, more particles are injected into the simulation domain, while there is less time for particles to diffuse vertically outside the core region while they flow downstream quite quickly. Hence, a non-zero average exit velocity results in a higher density at points inside the plume core and a lower density at points outside the core region.

Notice $\theta_{2}>\theta_{1}, 0<\theta_{2}<\pi / 2$ and $-\pi / 2<\theta_{1}<\pi / 2$, the number density and velocities at any point $(X, Y)$ in front of the slit can be derived. ${ }^{14}$ The final results are:

$$
\begin{aligned}
n(X, Y)= & \frac{\exp \left(-\beta U_{0}{ }^{2}\right)}{2 \pi}\left(\theta_{2}-\theta_{1}\right)+\frac{1}{4}\left(\operatorname{erf}\left(\sqrt{\beta} U_{0} \sin \theta_{2}\right)-\frac{\theta_{1}}{\left|\theta_{1}\right|} \operatorname{erf}\left(\sqrt{\beta} U_{0} \sin \left|\theta_{1}\right|\right)\right) \\
& +\frac{\sqrt{\beta / \pi}}{2} \int_{\theta_{1}}^{\theta_{2}} \exp \left(-\beta U_{0}{ }^{2} \sin ^{2} \theta\right) U_{0} \cos \theta \operatorname{erf}\left(\sqrt{\beta} U_{0} \cos \theta\right) d \theta \\
\frac{U(X, Y)}{\sqrt{2 R T_{0}}}= & \frac{\exp \left(-\beta U_{0}{ }^{2}\right)}{2 n \pi \sqrt{2 R T_{0}}}\left[\int_{\theta_{1}}^{\theta_{2}}\left(\frac{\sqrt{\pi / \beta}}{2} \exp \left(\beta U_{0}{ }^{2} \cos ^{2} \theta\right) \cos \theta\left(1+\operatorname{erf}\left(\sqrt{\beta} U_{0} \cos \theta\right)\right)\right) d \theta\right. \\
& +\frac{U_{0}\left(\theta_{2}-\theta_{1}\right)}{2}+\frac{U_{0}\left(\sin \left(2 \theta_{2}\right)-\sin \left(2 \theta_{1}\right)\right)}{4} \\
& \left.+\sqrt{\beta \pi} \int_{\theta_{1}}^{\theta_{2}}\left(U_{0}{ }^{2} \cos ^{3} \theta\left(1+\operatorname{erf}\left(\sqrt{\beta} U_{0} \cos \theta\right)\right) \exp \left(\beta U_{0}{ }^{2} \cos \theta\right)\right) d \theta\right]
\end{aligned}
$$

25 of 33

American Institute of Aeronautics and Astronautics 

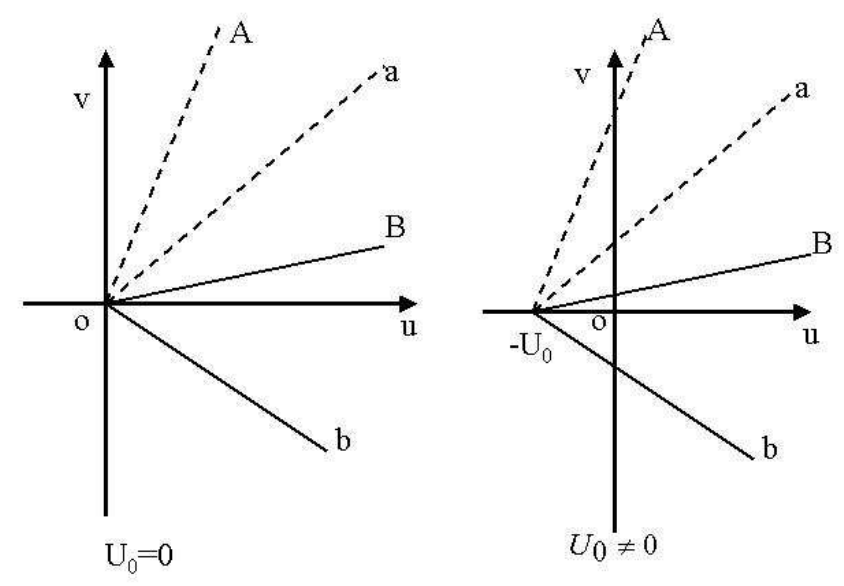

Figure 26. Effect on Velocity Space by the Average Exit Velocity.

$\frac{V(X, Y)}{\sqrt{2 R T_{0}}}=\frac{1}{4 \sqrt{\pi} n}\left[\exp \left(-\beta U_{0}^{2} \sin ^{2} \theta_{1}\right) \cos \theta_{1}\left(1+\operatorname{erf}\left(\sqrt{\beta} U_{0} \cos \theta_{1}\right)\right)-\exp \left(-\beta U_{0}^{2} \sin ^{2} \theta_{2}\right) \cos \theta_{2}\left(1+e r f\left(\sqrt{\beta} U_{0} \cos \theta_{2}\right)\right)\right]$

This set of solutions includes two types of factors: geometry factors represented by $\theta_{1}$ and $\theta_{2}$ and other factors involving complex nonlinear relations with $U_{0}$, the average exit velocity at the slit.

From Equations 54-56, it can be proved that: $n(X,-Y)=n(X, Y), U(X,-Y)=U(X, Y), V(X,-Y)=$ $-V(X, Y)$. These results can be obtained with aid from the fact that for points $(X, Y)$ and $(X,-Y)$, the integral domains in velocity space are symmetric about the u-axis.

It is also evident that these solutions are totally compatible with the previous solutions to Case 1 . When the average velocity is zero, this current set of general solutions degenerates consistently to the special solutions obtained for the first problem. Another interesting comparison is the quantities on the exit center and exit tip. Though it is difficult to obtain the exact values at these two points, the integral domain has some simple properties: on the exit center, the integral domain is from $-\frac{\pi}{2}$ to $\frac{\pi}{2}$, while on the exit tip, the integral domain is from 0 to $\frac{\pi}{2}$. Hence, it can be concluded from Equations $54-55$ that the number density at the exit tip is half of the value at the exit center, while the velocity along the $X$ direction has the same value.

Because this case has a non-zero average exit velocity, which is realistic for a real plume flow, it is worthy to investigate several kinds of approximations under special conditions, at least for the number density expressions. Equations 54 can be simplified under different conditions by removing the integral expressions and several simplifications are listed here for reference. In the far field, because $\tan \left(\theta_{2}-\theta_{1}\right)=\frac{\tan \theta_{2}-\tan \theta_{1}}{1+\tan \theta_{2} \tan \theta_{1}}$, hence, $\theta_{2}-\theta_{1}=\arctan \left(\frac{2 H X}{X^{2}+Y^{2}-H^{2}}\right) \approx \frac{2 H r \cos \alpha}{r^{2}-H^{2}}$, which is quite small, then:

$$
n(X, Y)=\frac{H r \cos \alpha}{r^{2}-H^{2}}\left[\sqrt{\frac{\beta}{\pi}}\left(1+\operatorname{erf}\left(\sqrt{\beta} U_{0} \cos \alpha\right)\right) \exp \left(-\beta U_{0}^{2} \sin ^{2} \alpha\right) U_{0} \cos \alpha+\frac{\exp \left(-\beta U_{0}^{2}\right)}{\pi}\right]
$$

Equations 54 and 57 indicate that there are cosine factors in the expression, quite consistent with the solutions obtained by Narasimha ${ }^{6}$ for flows through an orifice with high speed. Further, if the average velocity at the slit is large, the plume is confined to a small region close to the $\mathrm{X}$-axis. Hence, with a high average exit

26 of 33

American Institute of Aeronautics and Astronautics 
speed and a small $\alpha$ for points inside the core region, the results further take new formats:

$$
n(X, Y)=\frac{2 H X}{X^{2}+Y^{2}-H^{2}}\left(\frac{\exp \left(-\beta U_{0}^{2}\right)}{2 \pi}+\frac{\sqrt{\beta / \pi} U_{0} X}{\sqrt{X^{2}+Y^{2}}}\right)
$$

With a low average exit speed, since $\operatorname{erf}(x) \approx \frac{2 x}{\sqrt{\pi}}$ and $\exp (-x) \approx 1-x$,

$$
n(X, Y)=\frac{\theta_{2}-\theta_{1}}{2 \pi}+\frac{\sqrt{\beta / \pi} U_{0}\left(\sin \theta_{2}-\sin \theta_{1}\right)}{2}+\frac{\sin \left(2 \theta_{2}\right)-\sin \left(2 \theta_{1}\right)}{4 \pi} \beta U_{0}{ }^{2}
$$

\section{B. Numerical Simulations and Discussions}

Though the Equations 54-56 involve several integral terms that cannot be explicitly removed, numerical evaluations are convenient via a computer. The subroutine for the error function can be found in any numerical computing book. ${ }^{17}$ The simulation domain and mesh are the same as the first case discussed previously, and the collision function in MONACO is turned off.

The existence of nonzero average exit velocity has a significant effect on the flowfield. If the exit average velocity $U_{0}=0$, the boundary line between the flow field and a vacuum is the vertical line above the slit. When the average exit velocity at the exit increases, the boundary line begins to decline towards the plume core, though it still starts with the upper tip $(0, H)$. Hence, there will be a larger void region connected with point $(0, H)$ and residing above the slit. This presents a problem for evaluation of the analytical results because the number density is a component of the denominators in the velocities expressions, and in the analytical vacuum region, the numerical error may exceed the small analytical magnitudes of the number densities. For simulation results from the DSMC method, this is not a problem because the evolution of velocities does not involve a denominator of number density. For the analytical solutions to the sixth problem, an effective boundary line of $n / n_{0}=0.0001$ is introduced to represent the plume edge, and the comparisons are restricted to areas within this boundary. Figure 27 shows several such effective boundary lines with different average exit velocities at the slit. It indicates that as the average exit velocity increases, the plume region narrows because particles have less time to diffuse vertically. When the exit speed is high enough, the flow field is confined to a narrow region close to the plume core.

Figure 28 shows a comparison of number density contours with the DSMC results evaluated at $K n=20$. By turning off the collision function in the DSMC method, the results are expected to be close to free molecular. Generally the comparison shows almost identical results.

Figures 29 and 30 show the corresponding simulation results of velocity contours. The Knudsen number is set to 20 and the average exit velocity at the slit is the sonic speed. Due to the singularity problem mentioned earlier, the analytical results are not valid beyond the boundary line hence they are omitted here. Compared with Figures 3 and 4 from Case 1, it can be observed that the flow patterns are the same, including a narrow zone where exit effects dominate, straight contour lines in the far field and the same variation trends. Figures 3, 4, 29 and 30 strongly indicate that the solutions to these two flow problems are closely related.

Figure 31 shows a comparison of number density distribution along the plume centerline $Y=0$ and the slit tip $Y=H$. An average exit velocity $U_{0}=\sqrt{\gamma R T}$ results in a density value close to 0.9 at the exit center, which is illustrated on the starting point of one curve. Point $(0, H)$ is a singularity point and its number density value is close to half of the centerline value. The comparison of number density along line $Y=H$ indicates that in general the numerical and analytical results agree quite well. This plot also indicates that with this exit velocity, at downstream locations about $X=5 H$, these two number density distributions merge into one.

Figures 32 and 33 show comparisons of velocities along the centerline $Y=0$ and the slit tip $Y=H$. It is evident that the numerical results for velocity along the $Y$ direction have a perfect agreement.

Figures 34 and 35 compare different profiles of number densities along the plume centerline with different average exit velocities $U_{0}=0.1 \sqrt{\gamma R T_{0}}$ and $U_{0}=3 \sqrt{\gamma R T_{0}}$. The Knudsen numbers are set to 20 and these 


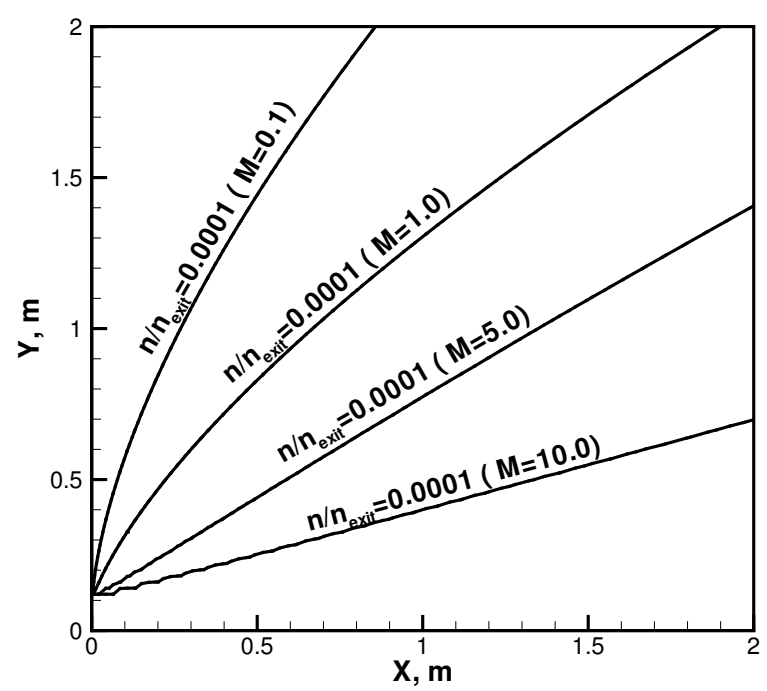

Figure 27. Case 6: Analytical Plume Boundary Lines $(H=0.1 m)$.

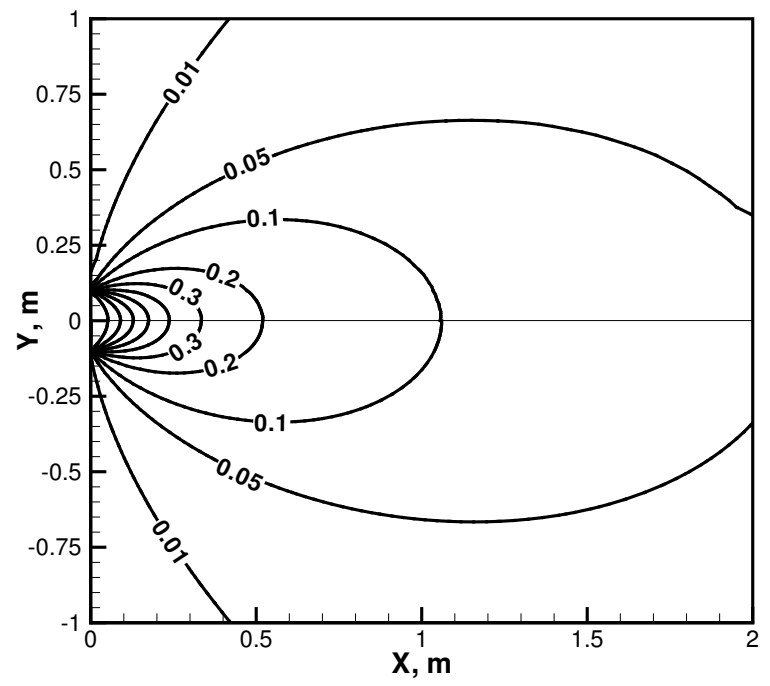

Figure 28. Case 6: Contours of $n(X, Y)\left(H=0.1 \mathrm{~m}, U_{0}=\sqrt{\gamma R T_{0}}\right.$, Top: DSMC, Bottom: Analytical).

28 of 33

American Institute of Aeronautics and Astronautics 


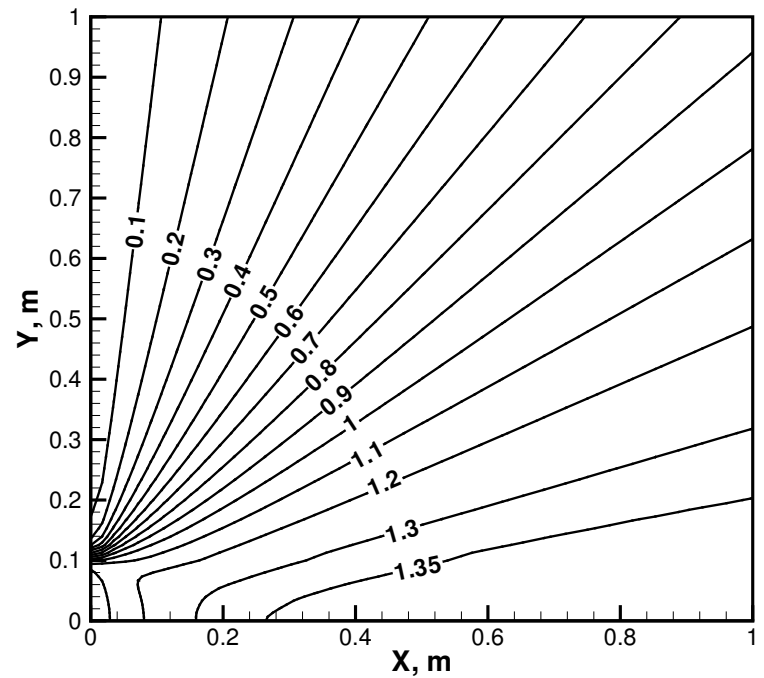

Figure 29. Case 6: Contours of $\frac{U(X, Y)}{\sqrt{2 R T_{0}}}$ (DSMC, $\left.H=0.1 \mathbf{m}, U_{0}=\sqrt{\gamma R T_{0}}\right)$.

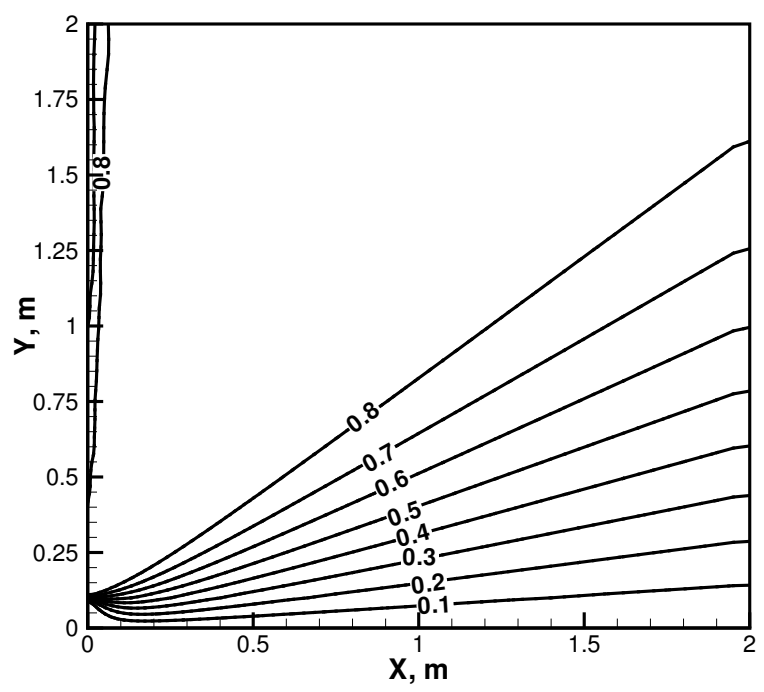

Figure 30. Case 6: Contours of $\frac{V(X, Y)}{\sqrt{2 R T_{0}}}$ (DSMC, $\left.H=0.1 \mathbf{m}, U_{0}=\sqrt{\gamma R T_{0}}\right)$.

29 of 33

American Institute of Aeronautics and Astronautics 


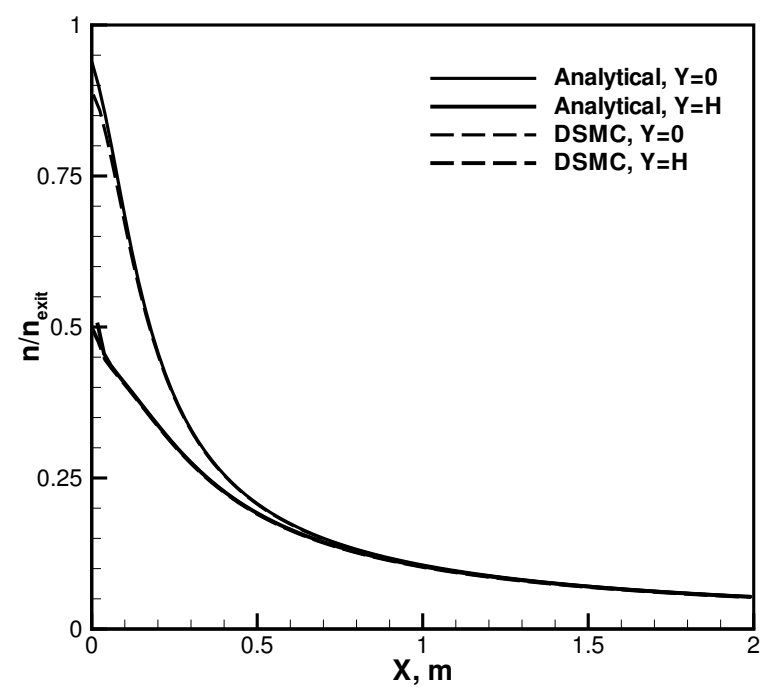

Figure 31. Case 6: Comparisons of Number Density along $Y=0$ and $Y=\mathbf{H}\left(U_{0}=\sqrt{\gamma R T_{0}}, H=0.1 \mathrm{~m}\right)$.

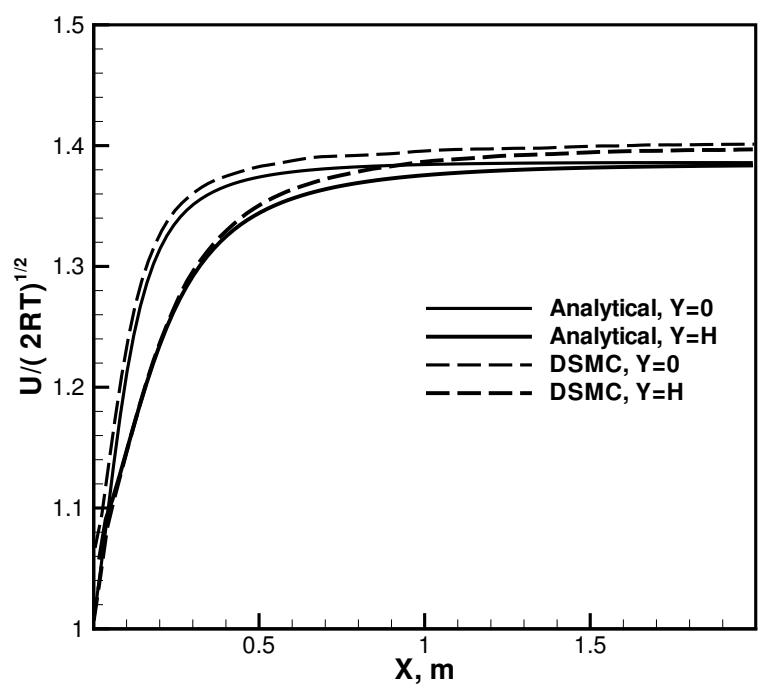

Figure 32. Case 6: Comparisons of $\frac{U(X, Y)}{\sqrt{2 R T_{0}}}$ along $Y=\mathbf{0}$ and $Y=\mathbf{H}\left(U_{0}=\sqrt{\gamma R T_{0}}, H=\mathbf{0 . 1} \mathbf{~ m}\right)$.

30 of 33

American Institute of Aeronautics and Astronautics 


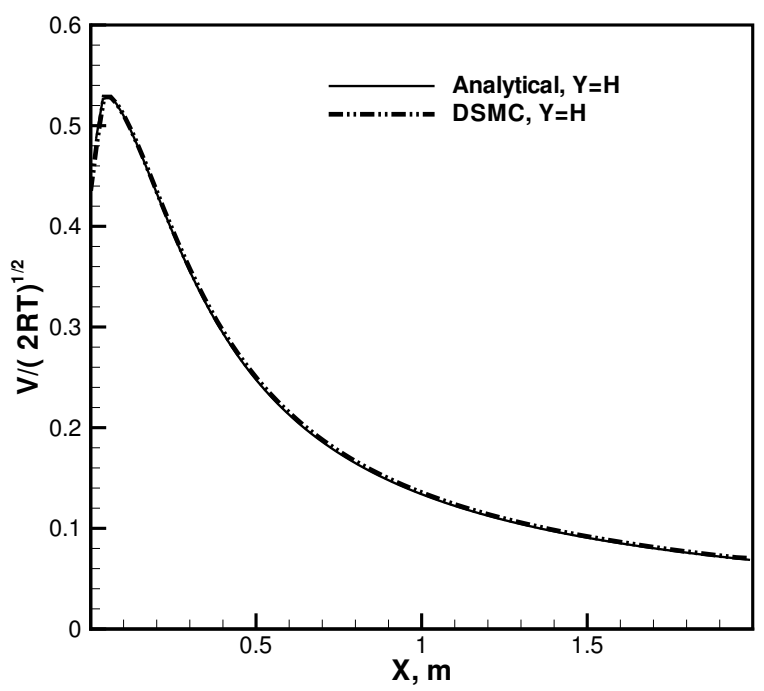

Figure 33. Case 6: Comparisons of $\frac{V(X, Y)}{\sqrt{2 R T_{0}}}$ along $Y=\mathbf{H}\left(U_{0}=\sqrt{\gamma R T}, H=\mathbf{0 . 1} \mathbf{~ m}\right)$.

distributions are obtained from different approximations, exact solution and numerical simulations. The comparisons clearly indicate that for both low speed and high speed:

1). The exact solutions agree with the DSMC results;

2). The low speed simplification agrees with the exact solution quite well for $U_{0}=0.1 \sqrt{\gamma R T_{0}}$;

3). Surprisingly, the high speed approximation and the far field approximation agree quite well for $U_{0}=0.1 \sqrt{\gamma R T_{0}}$ as well;

4). For $U_{0}=3 \sqrt{\gamma R T_{0}}$, after certain distances down stream of a few slit widths, the far field and the high speed approximations predict similar values to the exact analytical and numerical results.

It can be concluded that for number density distributions, over a wide range of average exit velocities, these approximations yield quite good agreement.

\section{Summary}

In this study, six fundamental free molecular flows out of an exit with different geometries were investigated analytically and validated by particle simulations. The important velocity-position relations, Equations $8,15,36$ and 52, provided one-to-one mapping relations between velocity spaces for a specific point in front of the exit and a point on the exit. These four relations validated Equation 7 and provided integral domains for all six problems.

This study yielded analytical exact solutions or exact expressions with detailed exit geometries. The first five cases belong to a category of true effusion problems with a zero average exit velocity. Their flowfields were associated with geometry factors and no collisions effects were considered. The last problem was of significant importance, because it is the case closest to realistic plume flows. The analytical results of the last problem indicated that the solutions were composed of two factors: one factor representing simple geometry relations and the other factor representing complex nonlinear effects from the average exit velocity at the slit. These results included accurate geometry factors, hence they were expected to yield more accurate results than existing models in the literature. These analytical results clearly captured all features of the 


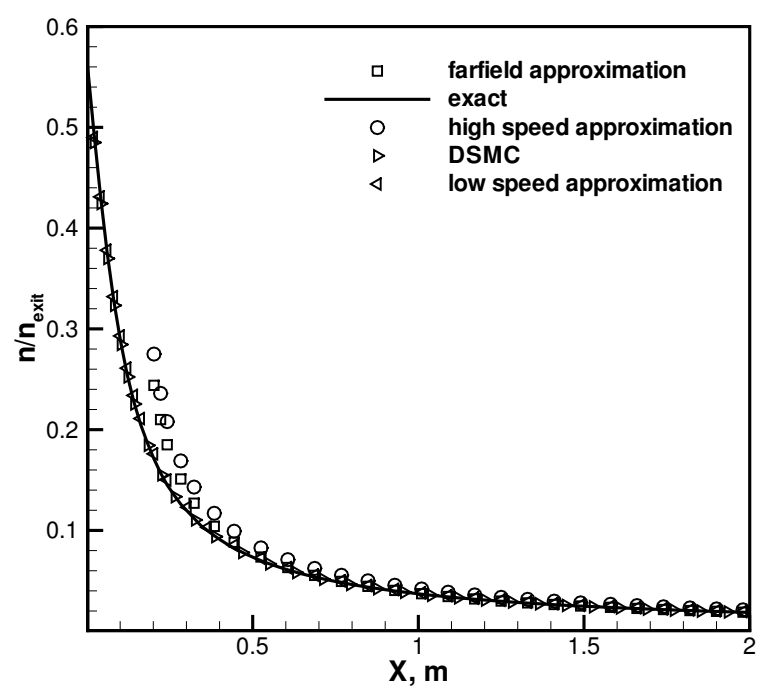

Figure 34. Case 6: Comparisons of Number Density along $\mathbf{Y}=\mathbf{0}\left(R=\mathbf{0 . 1} \mathbf{~ m}, U_{0}=0.1 \sqrt{\gamma R T_{0}}\right)$.

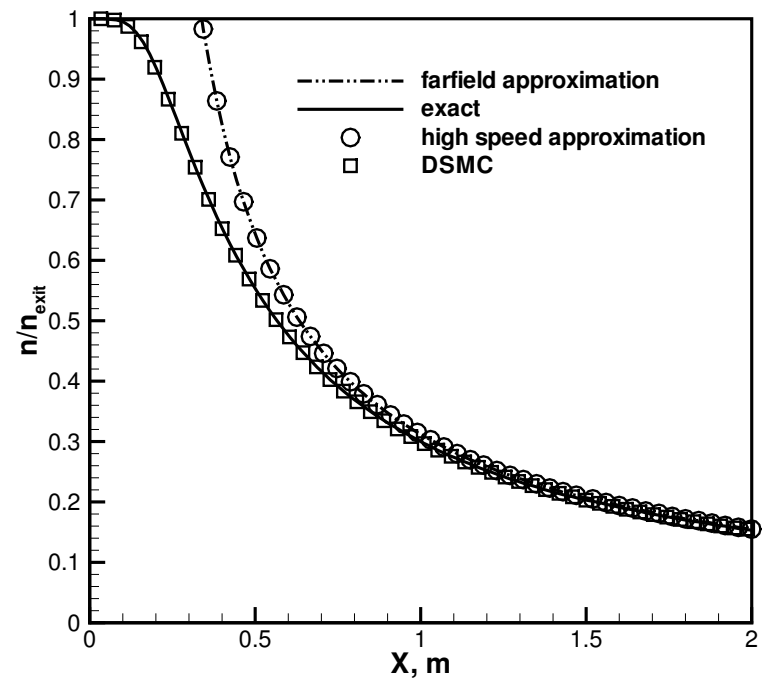

Figure 35. Case 6: Comparisons of Number Density along $\mathbf{Y}=\mathbf{0}\left(R=0.1 \mathbf{m}, U_{0}=3 \sqrt{\gamma R T_{0}}\right)$.

32 of 33

American Institute of Aeronautics and Astronautics 
whole flow field.

Several particle simulations were performed with the DSMC method to validate the analytical solutions obtained in this study, and in these simulations, the collision functions were turned off. Generally simulations for all these six cases yield good agreement with the analytical results, most of the results are essentially identical. It can be concluded that the general treatment of these six free molecular flows from different exit shapes are valid.

This study is an initial effort to provide analytical results to describe the free molecular plume flows from electric propulsion thrusters. It provided a solid basis for further analytical studies, such as plume flows out of a circular or annular thruster with a non-zero average velocity at the thruster exit.

\section{Acknowledgment}

This work was carried out and completed at the University of Michigan. The authors gratefully acknowledge funding for this work from the Air Force Office of Scientific Research under grant FA9550-05-1-0042.

\section{References}

${ }^{1}$ Aktas, O., Aluru, N. R. and Ravaioli, U., "Application of a parallel DSMC technique to predict flow characteristics in microfluidic filters", J. of Microelectromech. Sys. Vol. 10, pp.538-549, 2001.

${ }^{2}$ Jamison, A. J., Ketsdever, A. D. and Muntz, E. P., "Gas dynamic calibration of a nano-Newton thrust stand", Rev. Sci. Instrum. Vol. 73, pp.3629-3673, 2002.

${ }^{3}$ Szwemin, P., Szymanski, K. H. and Jousten, S., "Monte Carlo study of a new PTB primary standard for very low pressure", Metrologia, 36, No.1, pp.562-564, 1999.

${ }^{4}$ Liepmann, H. W., "Gas kinetics and gas dynamics of orifice flow", J. Fluid Mech., Vol. 10, pp.65-79,1961.

${ }^{5}$ Narasimha, R., "Orifice flow of high knudsen number", J. Fluid Mech. Vol.10, pp.371-384, 1961.

${ }^{6}$ Narasimha, R., "Collisionless expansion of gases into vacuum", J. Fluid Mech. Vol. 12, pp.294-308,1962.

${ }^{7}$ Narasimha, R., Some flow problems in rarefied gas dynamics. Ph.D. thesis, California Institute of Technology, Pasadena, 1961.

${ }^{8}$ Willis, D. R., "Mass flow through a circular orifice and a two-dimensional slit at high Knudsen numbers", J. Fluid Mech., 21, pp.21-32, 1965.

${ }^{9}$ Rotenberg, A. N and Weitzner, H., "Nearly Free Flow Through an Orifice", Physics of Fluids, Vol. 12, 1573-1581, 1969.

${ }^{10}$ Sharipov, F., "Numerical Simulation of Rarefied Gas Flow Through a Thin Orifice", J. Fluid Mech. , Vol. 518, pp.35-60, 2004.

${ }^{11}$ Bird, G. A., Molecular gas dynamics and the direct simulation of gas flows. Oxford University Press, New York, 1994.

${ }^{12}$ Gombosi, T. I., Gaskinetic theory. Cambridge University Press, New York, 1994.

${ }^{13}$ Vincenti, W. G. and Kruger, C. H. Kruger, Introduction to physical gas dynamics. Krieger Publishing, Malabar, Florida, 1986.

${ }^{14} \mathrm{Cai}$, C., Theoretical and numerical studies of plume flows in vacuum chambers. Ph.D. thesis, Dept. of Aerospace Engineering, University of Michigan, Ann Arbor, Michigan, October, 2005.

${ }^{15}$ Dietrich, S. and Boyd, I. D., "Scalar and parallel optimized implementation of the direct simulation Monte Carlo Method", J. Comput. Physics, 126, 328-342, 1996.

${ }^{16}$ Patterson, G.N., "Introduction to the Kinetic Theory of Gas Flows," Univ. Toronto Press, Toronto, 1971.

${ }^{17}$ Press, W. H., Vetterling, S. A., Flannery, W. T. and Flannery, B. P., Numerical recipes in C. Cambridge University Press, New York, 1994.

33 of 33

American Institute of Aeronautics and Astronautics 